\title{
Random Measurable Selections
}

\author{
Jean Goubault-Larrecq ${ }^{1} \quad$ Roberto Segala ${ }^{2}$ \\ 1 ENS Cachan goubault@lsv.ens-cachan.fr \\ 2 Università di Verona roberto.segala@univr.it
}

\begin{abstract}
We make the first steps towards showing a general "randomness for free" theorem for stochastic automata. The goal of such theorems is to replace randomized schedulers by averages of pure schedulers. Here, we explore the case of measurable multifunctions and their measurable selections. This involves constructing probability measures on the measurable space of measurable selections of a given measurable multifunction, which seems to be a fairly novel problem. We then extend this to the case of IT automata, namely, non-deterministic (infinite) automata with a history-dependent transition relation. Throughout, we strive to make our assumptions minimal.
\end{abstract}

\section{Introduction}

This paper grew out of an attempt at proving a "randomness for free" type theorem [5] for stochastic automata [4]. We present the first steps in this direction.

A stochastic automaton is a transition system on a measurable space $Q$ of states. When in state $q \in Q$, we have access to a set $\theta(q)$ of fireable transitions, from which we choose non-deterministically. A transition is a pair $(a, \mu)$ of an action $a$, from a fixed measurable space $L$, and of a probability measure $\mu$ on $Q$. Once we have chosen such a transition, we pick the next state $q^{\prime}$ at random with respect to $\mu$, and proceed. There are two ways to resolve the non-deterministic choice of a transition $(a, \mu) \in \theta(q)$. A pure scheduler $\sigma$ is a function that maps each path $w=q_{0} a_{1} q_{1} \cdots a_{n} q_{n}$ of states and actions seen so far to an element $\sigma(w)$ of $\theta\left(q_{n}\right)$ (or to a special termination constant $\perp$ ). A randomized scheduler $\eta$ instead maps $w$ to a (sub)probability measure concentrated on $\theta\left(q_{n}\right)$, thereby drawing the transition at random as well. In each case, given a scheduler $\eta$, the stochastic automaton behaves as a purely probabilistic transition system, and one can define the probability $P_{\eta}(\mathcal{E})$ that the automaton will follow a path that lies in the measurable set $\mathcal{E}$.

The "randomness for free" question we envision is as follows: given a randomized scheduler $\eta$, and a measurable set $\mathcal{E}$ of paths, can we find two pure schedulers $\sigma^{-}, \sigma^{+}$such that $P_{\sigma^{-}}(\mathcal{E}) \leq P_{\eta}(\mathcal{E}) \leq P_{\sigma^{+}}(\mathcal{E})$ ? This has a number of important applications, and was solved positively by Chatterjee, Doyen et al. [5, Section 4], in the case that $Q$ and $L$ are finite. In general, we consider the following more general question: given a randomized scheduler $\eta$, and a measurable payoff function $h$ from paths to $\mathbb{R}^{+}$, can we find two pure schedulers 
$\sigma^{-}, \sigma^{+}$such that $\int_{\omega} h(\omega) d P_{\sigma^{-}} \leq \int_{\omega} h(\omega) d P_{\eta} \leq \int_{\omega} h(\omega) d P_{\sigma^{+}}$? This includes the previous question, by taking the characteristic function $\chi_{\mathcal{E}}$ of $\mathcal{E}$ for $h$.

Beware that one cannot reasonably ask for the existence of a pure scheduler $\sigma$ such that $P_{\sigma}(\mathcal{E})=P_{\eta}(\mathcal{E})$. For example, let $Q=\{0,1,2\}, L=\{*\}, \theta(0)=$ $\left\{t_{1}=\left(*, \delta_{1}\right), t_{2}=\left(*, \delta_{2}\right)\right\}$ (where $\delta_{x}$ is the Dirac mass at $\left.x\right), \theta(1)=\theta(2)=\emptyset$. There are only two pure schedulers, one that reaches state 1 with probability 1 , while the other reaches 2 with probability 1 . But one can reach 1 with arbitrary probability $p$ using the randomized scheduler $\eta(0)=p \delta_{t_{1}}+(1-p) \delta_{t_{2}}$.

While Chatterjee, Doyen et al. are concerned with finite state and action spaces, infinite spaces are useful as well: in modeling timed probabilistic and non-deterministic transition systems [7, Example 1.1], or devices that interact with the physical world $[6,1]$, where each state includes information about time, position, impulse and other real-valued data, for example. Considering his publication record (see for example [16] on labeled Markov processes, or [10] on Markov decision processes), Prakash would probably be the first to approve us.

Our initial aim was to prove such "randomness for free" theorems for general stochastic automata. During the year 2008, we found very complex arguments that proved only very weak versions of what we were after. We were on the wrong path. On the opposite, Chatterjee, Doyen et al. [5, Section 4] used a simple idea: draw pure schedulers $\sigma$ themselves at random, with respect to some measure $\varpi$, designed so that $P_{\eta}(\mathcal{E})=\int_{\sigma} P_{\sigma}(\mathcal{E}) d \varpi$. The claim then follows by standard integration arguments (Fact 1 below).

The probability measure $\varpi$ on pure schedulers has an intuitive description, too: we merely choose the transition $\sigma(w)$ at random with respect to probability $\eta(w)$, where $\eta$ is our given randomized scheduler, for each finite path $w$, independently. Now this is the source of typical measure-theoretic complications. First, we must force our pure schedulers to be measurable. Otherwise, $\int_{\sigma} P_{\sigma}(\mathcal{E}) d \varpi$, and in fact already $P_{\sigma}$, makes no sense. Second, we need to make clear what the intended $\sigma$-algebra is on the space of all pure schedulers. If we don't have any, nothing of the above makes any sense either. Third, what does it mean to draw $\sigma(w)$ for each $w$ independently? The sheer fact that $\sigma$ is measurable must enforce at least some moderate amount of non-independence.

Chatterjee, Doyen et al. did not need to address these issues: on finite state and action spaces, all pure schedulers are measurable, and the problems above disappear. Going to infinite spaces of states and actions requires extra effort.

While we were writing this paper, we soon realized that we would have to choose between: (1) solving the full question, by applying all the required measure-theoretic clout if necessary, possibly making it so complex that nobody would understand it; or (2) solving a few restricted cases, showing a few elegant proof tricks along the way. It should be clear that (2) was a better choice. We hope that Prakash will appreciate the techniques, if not the results.

That is, we shall be content to solve the problem in the special case of IT automata, namely, stochastic automata with a trivial action space, no option for termination, and no random choice at all: randomness will be induced by the randomized scheduler only. We shall deal with the general case in another paper. 
The plan of the paper is as follows. We recapitulate some required basic measure theory in Section 2. In Section 3, we define a $\sigma$-algebra on the set $\operatorname{Sel}(F)$ of measurable selections of a given multifunction $F$ - these are just what we have called pure schedulers, for the transition relation $F$ of a stochastic automaton without probabilistic choice; and we show that any randomized scheduler $g$ defines a canonical probability measure $\varpi_{g}$ on $\operatorname{Sel}(F)$ such that, for every $x$, drawing a point at random with probability $g(x)$ gives the same result as drawing a measurable selection $f$ with probability $\varpi_{g}$ and computing $f(x)$ (Proposition 1). This is the most important construction of the paper. In Section 4, we extend this result from random measurable selections to random measurable pure schedulers of IT automata. Although the setting looks extremely similar, there is no hope of reusing the previous result. Instead, we use similar proof techniques, but with a more complex implementation. We conclude in Section 5.

\section{Basics on Measure Theory}

A $\sigma$-algebra on a set $X$ is a family of subsets that is closed under complement and countable unions. We shall write $\bar{A}$ for the complement of $A$ in $X$. A pair $X=\left(|X|, \Sigma_{X}\right)$ of a set $|X|$ and a $\sigma$-algebra $\Sigma_{X}$ on $|X|$ is a measurable space, and the elements of $\Sigma_{X}$ are called the measurable subsets of $X$. We shall sometimes write $X$ instead of $|X|$ to avoid pedantry.

Given any family $\mathcal{F}$ of subsets of a set $A$, there is a smallest $\sigma$-algebra that contains the elements of $\mathcal{F}$. This is called the $\sigma$-algebra generated by $\mathcal{F}$. We shall sometimes refer to the elements of $\mathcal{F}$ as the basic measurable subsets of this $\sigma$-algebra, despite some ambiguity. One example is $\mathbb{R}$ with its Borel $\sigma$-algebra, generated by intervals. Another one is the product $X_{1} \times X_{2}$ of two measurable spaces is $\left(\left|X_{1}\right| \times\left|X_{2}\right|, \Sigma_{X_{1}} \otimes \Sigma_{X_{2}}\right)$, whose basic measurable subsets are the rectangles $E_{1} \times E_{2}, E_{1} \in \Sigma_{X_{1}}, E_{2} \in \Sigma_{X_{2}}$. In general, the $\sigma$-algebra on the product of an arbitrary family of measurable spaces $\left(X_{i}\right)_{i \in I}$ is the one generated by the subsets $\pi_{i}^{-1}(E)$ where $E \in \Sigma_{X_{i}}, i \in I$, and $\pi_{i}: \prod_{i \in I}\left|X_{i}\right| \rightarrow\left|X_{i}\right|$ is the usual projection onto coordinate $i$.

The coproduct $X_{1}+X_{2}$ of two measurable spaces $X_{1}, X_{2}$ is simpler: $\left|X_{1}+X_{2}\right|$ is the disjoint union of $\left|X_{1}\right|$ and $\left|X_{2}\right|$, and $\Sigma_{X_{1}+X_{2}}$ consists of unions $E_{1} \cup E_{2}$ of a measurable subset $E_{1}$ of $X_{1}$ and of a measurable subset $E_{2}$ of $X_{2}$. This construction generalizes to countable coproducts $\sum_{n \in \mathbb{N}} X_{n}$ in the obvious way.

A measurable map $f: X \rightarrow Y$ is one such that $f^{-1}(E) \in \Sigma_{X}$ for every $E \in \Sigma_{Y}$. If $\mathcal{F}$ generates $\Sigma_{Y}$, it is enough to check that $f^{-1}(E) \in \Sigma_{X}$ for every $E$ in $\mathcal{F}$ to establish the measurability of $f$.

A measure $\mu$ on $X$ is a map from $\Sigma_{X}$ to $\mathbb{R}^{+} \cup\{+\infty\}$ that is countably additive $\left(\mu(\emptyset)=0\right.$, and $\mu\left(\bigcup_{n \in \mathbb{N}} E_{n}\right)=\sum_{n \in \mathbb{N}} \mu\left(E_{n}\right)$ for every countable family of disjoint measurable subsets $\left.E_{n}\right)$. A probability measure is one such that $\mu(X)=1$. The Dirac mass at $x, \delta_{x}$, is the probability measure defined by $\delta_{x}(E)=1$ if $x \in E$, 0 otherwise.

A measure $\mu$ is concentrated on a measurable subset $A$ of $X$ if and only if $\mu(X \backslash A)=0$. For example, if $X$ is finite and $\Sigma_{X}=\mathbb{P}(|X|)$, then $\mu=\sum_{x \in X} a_{x} \delta_{x}$ 
is concentrated on $\left\{x \in X \mid a_{x} \neq 0\right\}$. Any subset $A$ (even non-measurable) of $|X|$ gives rise to a measurable subspace, again written $A$, with $\Sigma_{A}=\{A \cap B \mid$ $\left.B \in \Sigma_{X}\right\}$. If $A$ is measurable, one can define the restriction $\mu_{\mid A}$ of $\mu$ to the subspace $A$, by $\mu_{\mid A}(B)=\mu(B)$ for every $B \in \Sigma_{A}$. If $\mu$ is a probability measure that is concentrated on $A$, then $\mu_{\mid A}$ is also a probability measure.

There is a standard notion of integral of measurable maps $h: X \rightarrow \mathbb{R}^{+}$with respect to a measure $\mu$ on $X$, which we write $\int_{x \in X} h(x) d \mu$. Other notations found in the literature are $\int_{x \in X} h(x) \mu(d x)$ or $\langle h, \mu\rangle$. We shall also use the notation $\int_{x \in X} d \mu h(x)$, especially when $h(x)$ is a long formula, as in $\int_{x_{1} \in E_{1}} d \mu_{1} \int_{x_{2} \in E_{2}} d \mu_{2}$ $h\left(x_{1}, x_{2}\right)$. Writing $\chi_{E}$ for the characteristic map of a measurable subset $E$ of $X$, $\int_{x \in X} \chi_{E}(x) d \mu$ equals the measure $\mu(E)$ of $E$.

Given a map $f: A \rightarrow|X|$ (not necessarily measurable) where $X$ is a measurable space and $A$ is a set, the family $\left(f^{-1}(E)\right)_{E \in \Sigma_{X}}$ is a $\sigma$-algebra on $A$, called the $\sigma$-algebra induced by $f$ on $A$. When $f$ is the inclusion map of a subset $A$ of $|X|$, we retrieve the subspace $\sigma$-algebra $\Sigma_{A}$.

If $\mu$ is concentrated on a measurable subset $A$ of $X$, then $\int_{x \in X} g(x) d \mu=$ $\int_{x \in A} g(x) d \mu_{\mid A}$, where $A$ is considered as a subspace of $X$ on the right-hand side.

We write $\mathcal{P}(X)$ for the space of all probability measures on $X$, with the weak $\sigma$-algebra, generated by the subsets $[E>r]=\{\mu \in|\mathcal{P}(X)| \mid \mu(E)>r\}$. One can equate $\mu \in \mathcal{P}(X)$ with a vector of real numbers $(\mu(E))_{E \in \Sigma_{X}}$, i.e., with an element of the measurable space $\mathbb{R}^{\Sigma_{X}}$. The weak $\sigma$-algebra is nothing else than the $\sigma$-algebra induced by the inclusion of $|\mathcal{P}(X)|$ into the product $\mathbb{R}^{\Sigma_{X}}$.

Given a measurable map $f: X \rightarrow Y$, and a measure $\mu$ on $X$, the formula $f[\mu]\left(E^{\prime}\right)=\mu\left(f^{-1}\left(E^{\prime}\right)\right)$ defines a measure $f[\mu]$ on $Y$, called the image measure of $\mu$ by $f$. For any measurable $h: Y \rightarrow \mathbb{R}^{+}$, the following change of variables formula holds [2, Theorem 16.13]:

$$
\int_{y \in Y} h(y) d f[\mu]=\int_{x \in X}(h \circ f)(x) d \mu .
$$

More trivially, the function $\mathfrak{e}_{X}: X \rightarrow \mathcal{P}(X)$ that sends $x$ to the Dirac mass $\delta_{x}$ is measurable. These facts assemble to define the so-called Giry monad [11, Section 1], of which $\mathfrak{e}$ is the unit. (More precisely, one of the two Giry monads.) Prakash stressed the importance of this monad in [14] — probably the one paper that popularized it.

Finally, we shall use the following well-known fact near the end of the paper.

Fact 1 For every integrable map $h: X \rightarrow \mathbb{R}$ on a measurable space $X$, for every $a \in \mathbb{R}$, if $\int_{x \in X} h(x) d \mu=a$ for some probability measure $\mu$ on $X$, then there are points $x^{-}, x^{+} \in|X|$ such that $h\left(x^{-}\right) \leq a \leq h\left(x^{+}\right)$.

Indeed, if $x^{-}$did not exist, say, then $h(x)>a$ for every $x \in|X|$. Let $A_{n}=$ $h^{-1}(a+1 / n,+\infty)$ for every non-zero natural number $n$ : so $|X|=\bigcup_{n \geq 1} A_{n}$. Since $h(x) \geq a$ for every $x \in|X|$ and $h(x) \geq a+1 / n$ if additionally $x \in A_{n}$, $a=\int_{x \in X} h(x) d \mu \geq a+1 / n \mu\left(A_{n}\right)$, so $\mu\left(A_{n}\right)=0$. A consequence of $\sigma$-additivity is that the measure of the union of a countable chain of measurable subsets is the sup of their measures, so $1=\mu(|X|)=\sup _{n=1}^{+\infty} \mu\left(A_{n}\right)=0$ : contradiction. 
Carathéodory's measure extension theorem. The following measure extension theorem, due to Carathéodory, was singled out as "a very useful type of theorem" by Prakash [15, Theorem 18]; see also [2, Theorem 11.3].

A semiring $\mathcal{A}$ on a set $\Omega$ is a collection of subsets of $\Omega$ that contains the empty set, is closed under binary intersections, and such that the difference $A \backslash B$ of any two sets $A, B \in \Omega$ can be written as a finite union of elements of $\mathcal{A}$. A map $\mu$ is countably subadditive on $\mathcal{A}$ if and only if for every countable disjoint family of elements $A_{n}$ of $\mathcal{A}$ whose union $A$ is in $\mathcal{A}, \mu(A) \leq \sum_{n \in \mathbb{N}} \mu\left(A_{n}\right)$.

Theorem 1 (Carathéodory). Let $\Omega$ be a set, and $\mathcal{A}$ be a semiring on $\Omega$. Every function $\mu: \mathcal{A} \rightarrow[0,+\infty]$ such that $\mu(\emptyset)=0$, that is finitely additive and countably subadditive on $\mathcal{A}$, extends to a measure on the $\sigma$-algebra generated by $\mathcal{A}$. In particular, this is so if $\mu(\emptyset)=0$ and $\mu$ is countably additive on $\mathcal{A}$.

A typical application is $\Omega=\mathbb{R}, \mathcal{A}$ is the semiring of all half-closed intervals $[a, b)$, and $\mu[a, b)=b-a$, leading to Lebesgue measure.

The Ionescu-Tulcea Theorem. Let $Q_{n}, n \in \mathbb{N}$, be countably many measurable spaces, and assume countably many measurable maps $g_{n}: \prod_{i=0}^{n-1} Q_{i} \rightarrow \mathcal{P}\left(Q_{n}\right)$. One can think of $Q_{n}$ as the space of all possible states of a probabilistic transition system at (discrete) time $n \in \mathbb{N}$. Given that at time $n$ we have gone through states $q_{0} \in Q_{0}, q_{1} \in Q_{1}, \ldots, q_{n-1} \in Q_{n-1}, g_{n}\left(q_{0}, q_{1}, \cdots, q_{n-1}\right)$ is a probability distribution along which we draw the next state $q_{n}$. The following Ionescu-Tulcea Theorem states that these data define a unique probability measure on infinite paths $q_{0}, q_{1}, \cdots, q_{n-1}, \cdots$ :

Theorem 2 (Ionescu-Tulcea). Let $Q_{n}, n \in \mathbb{N}$, be measurable spaces, and $g_{*}=\left(g_{n}: \prod_{i=0}^{n-1} Q_{i} \rightarrow \mathcal{P}\left(Q_{n}\right)\right)_{n \geq 1}$ be measurable maps. For every $q_{0} \in Q_{0}$, there is a unique probability measure $P_{g_{*}}\left(q_{0}\right)$ on $\prod_{i=0}^{+\infty} Q_{i}$ such that:

$P_{g_{*}}\left(q_{0}\right)\left(\prod_{i=0}^{n} E_{i} \times \prod_{i=n+1}^{+\infty}\left|Q_{i}\right|\right)=\chi_{E_{0}}\left(q_{0}\right) \int_{q_{1} \in E_{1}} d g_{1}\left(q_{0}\right) \int_{q_{2} \in E_{2}} d g_{2}\left(q_{0} q_{1}\right) \cdots \int_{q_{n} \in E_{n}} d g_{n}\left(q_{0} q_{1} \cdots q_{n-1}\right)$.

Moreover, $P_{g_{*}}$ defines a measurable map from $Q_{0}$ to $\mathcal{P}\left(\prod_{i=0}^{+\infty} Q_{i}\right)$.

We consider tuples $\left(q_{0}, q_{1}, q_{2}, \cdots, q_{n-1}\right)$ as words, and accordingly write them as $q_{0} q_{1} q_{2} \cdots q_{n-1}$. The notation $\int_{q_{i} \in E_{i}} d \mu_{i} h\left(q_{i}\right)$ (where $\mu_{i}=g_{i}\left(q_{0} q_{1} \cdots q_{i-1}\right)$ above) stands for $\int_{q_{i} \in Q_{i}} d \mu_{i} \chi_{E_{i}}\left(q_{i}\right) h\left(q_{i}\right)$, and the rightmost integral in Theorem 2 is an integral of the constant 1, which is standardly omitted-i.e., the rightmost integral is $\int_{q_{n} \in Q_{n}} \chi_{E_{n}}\left(q_{n}\right) d g_{n}\left(q_{0} q_{1} q_{2} \cdots q_{n-1}\right)=g_{n}\left(q_{0} q_{1} q_{2} \cdots q_{n-1}\right)\left(E_{n}\right)$.

There are several small variations on the Ionescu-Tulcea Theorem. Our version is Giry's [11, Theorem 3], except for the fact that Giry considers more general ordinal-indexed sequences of measurable spaces. We will not require that.

The following is needed in the proof of Lemma 2. Lemma 2 looks perfectly obvious, yet requires some effort to prove. Measure theory is full of these. 
Lemma 1. Under the assumptions of Theorem 2, for every $n \in \mathbb{N}$, and for every measurable map $h: \prod_{i=0}^{n} Q_{i} \rightarrow \mathbb{R}^{+}$, for every $q \in Q_{0}$,

$$
\begin{aligned}
& \int_{q_{0} q_{1} \cdots \in \prod_{i=0}^{+\infty} Q_{i}} h\left(q_{0} q_{1} \cdots q_{n}\right) d P_{g_{*}}(q) \\
= & \int_{q_{1} \in Q_{1}} d g_{1}(q) \int_{q_{2} \in Q_{2}} d g_{2}\left(q q_{1}\right) \cdots \int_{q_{n} \in Q_{n}} d g_{n}\left(q q_{1} q_{2} \cdots q_{n-1}\right) h\left(q q_{1} q_{2} \cdots q_{n-1} q_{n}\right) .
\end{aligned}
$$

Proof. This is true for functions $h$ of the form $\chi_{\prod_{i=0}^{n} E_{i}}, E_{i} \in \Sigma_{Q_{i}}$, as one can check by using (2). Let $S$ be the set of measurable subsets $\mathcal{E}$ of $\prod_{i=0}^{n} Q_{i}$ such that (3) holds for $h=\chi_{\mathcal{E}}$, i.e., such that $P_{g_{*}}(q)(\mathcal{E})=\int_{q_{1} \in Q_{1}} d g_{1}(q) \int_{q_{2} \in Q_{2}} d g_{2}\left(q q_{1}\right) \cdots$ $\int_{q_{n} \in Q_{n}} d g_{n}\left(q q_{1} q_{2} \cdots q_{n-1}\right) \chi_{\mathcal{E}}\left(q q_{1} q_{2} \cdots q_{n-1} q_{n}\right) . S$ contains all the rectangles, is closed under complements (using $\chi_{\overline{\mathcal{E}}}=1-\chi_{\mathcal{E}}$ ), and under countable disjoint unions. For the latter, consider countably disjoint elements $\mathcal{E}_{m}, m \in \mathbb{N}$, of $S$, let $\mathcal{E}=\bigcup_{m \in \mathbb{N}} \mathcal{E}_{m}$, and realize that $\chi_{\mathcal{E}}=\sup _{m \in \mathbb{N}} \sum_{k=0}^{m} \chi_{\mathcal{E}_{k}}$. The Monotone Convergence Theorem [2, Theorem 16.2] states that integrals of non-negative real functions commute with pointwise suprema of countable chains, so $P_{g_{*}}(q)(\mathcal{E})=$ $\sup _{m \in \mathbb{N}} \sum_{k=0}^{m} \int_{q_{1} \in Q_{1}} d g_{1}(q) \int_{q_{2} \in Q_{2}} d g_{2}\left(q q_{1}\right) \cdots \int_{q_{n} \in Q_{n}} d g_{n}\left(q q_{1} q_{2} \cdots q_{n-1}\right) \chi_{\mathcal{E}_{k}}\left(q q_{1}\right.$ $\left.q_{2} \cdots q_{n-1} q_{n}\right)=\sum_{m=0}^{+\infty} P_{g_{*}}(q)\left(\mathcal{E}_{m}\right)$. It follows that $S$ is a $\sigma$-algebra containing the rectangles, and therefore contains $\Sigma_{\prod_{i=0}^{n} Q_{i}}$.

It follows easily that (3) holds for step functions $h$, i.e., when $h$ is of the form $\sum_{k=0}^{m} a_{k} \chi_{\mathcal{E}_{k}}, m \in \mathbb{N}, a_{k} \in \mathbb{R}^{+}, \mathcal{E}_{k}$ measurable. Since every measurable map $h: \prod_{i=0}^{n} Q_{i} \rightarrow \mathbb{R}^{+}$is the pointwise supremum of a countable chain of step functions (namely $h_{m}=\sum_{k=1}^{m 2^{m}} k / 2^{m} \chi_{h^{-1}\left(k / 2^{m},+\infty\right)}, m \in \mathbb{N}$ ), (3) follows by the Monotone Convergence Theorem.

Consider now any family of measurable subsets $\mathcal{E}_{n}$ of $\prod_{i=0}^{n-1} Q_{i} \times Q_{n}, n \geq 1$, and assume that for all $q_{0}, q_{1}, \ldots, q_{n-1}, g_{n}\left(q_{0} q_{1} \cdots q_{n-1}\right)$ draws $q_{n}$ at random so that $q_{0} q_{1} \cdots q_{n-1} q_{n}$ is in $\mathcal{E}_{n}$. It seems obvious that what we shall get in the end is an infinite path $q_{0} q_{1} \cdots q_{n} \cdots$ such that every finite prefix $q_{0} q_{1} \cdots q_{n}$ is in $\mathcal{E}_{n}$. This actually needs a bit of proof. Given a measurable subset $E$ of a product $A \times B$, and $a \in|A|$, the vertical cut $E_{\mid a}$ is the set $\{b \in|B| \mid(a, b) \in E\}$. This is measurable as soon as $E$ is [2, Theorem $18.1(i)]$.

Lemma 2. Under the assumptions of Theorem 2, let $\mathcal{E}_{n}$ be measurable subsets of $\prod_{i=0}^{n-1} Q_{i} \times Q_{n}, n \geq 1$, and assume that for all $q_{0} q_{1} \cdots q_{n-1} \in \prod_{i=0}^{n-1} Q_{i}$, $g_{n}\left(q_{0} q_{1} \cdots q_{n-1}\right)$ is concentrated on $\left(\mathcal{E}_{n}\right)_{\mid q_{0} q_{1} \cdots q_{n-1}}$.

For every $q \in Q_{0}, P_{g_{*}}(q)$ is concentrated on the set Path $\mathcal{E}_{\mathcal{E}}$ of infinite paths whose finites prefixes $q_{0} q_{1} \cdots q_{n}$ are in $\mathcal{E}_{n}$ for every $n \geq 1$. If additionally $\{q\}$ is measurable in $Q_{0}$, then $P_{g_{*}}(q)$ is concentrated on the set Path $\mathcal{E}(q)$ of those infinite paths in Path $_{\mathcal{E}}$ such that $q_{0}=q$.

Proof. First, Path $\mathcal{E}_{\mathcal{E}}$ is measurable, as a countable intersection of measurable subsets $\mathcal{E}_{n} \times \prod_{i=n+1}^{+\infty}\left|Q_{i}\right|$. Since $g_{n}\left(q_{0} q_{1} \cdots q_{n-1}\right)$ is concentrated on $\left(\mathcal{E}_{n}\right)_{\mid q_{0} q_{1} \cdots q_{n-1}}$, and the complement of the latter in $\left|Q_{n}\right|$ is $\left(\overline{\mathcal{E}_{n}}\right)_{\mid q_{0} q_{1} \cdots q_{n-1}}, \int_{q_{n} \in Q_{n}} \chi_{\left(\overline{\mathcal{E}_{n}}\right)_{\mid q_{0} q_{1} \cdots q_{n-1}}}$ 
$d g_{n}\left(q_{0} q_{1} \cdots q_{n-1}\right)=g_{n}\left(q_{0} q_{1} \cdots q_{n-1}\right)\left(\left(\overline{\mathcal{E}_{n}}\right)_{\mid q_{0} q_{1} \cdots q_{n-1}}\right)=0$. By taking $h=\chi_{\overline{\mathcal{E}_{n}}}$ in (3), and realizing that $\chi_{\overline{\mathcal{E}_{n}}}\left(q_{0} q_{1} \cdots q_{n-1} q_{n}\right)=\chi_{\left(\overline{\mathcal{E}_{n}}\right)_{\mid q_{0} q_{1} \cdots q_{n-1}}}\left(q_{n}\right)$, we obtain that $\int_{q_{0} q_{1} \cdots \in \prod_{i=0}^{+\infty} Q_{i}} \chi \overline{\mathcal{E}_{n}}\left(q_{0} q_{1} \cdots q_{n}\right) d P_{g_{*}}(q)=0$. In other words, the $P_{g_{*}}(q)$-measure of the complement of $\mathcal{E}_{n} \times \prod_{i=n+1}^{+\infty}\left|Q_{i}\right|$ is zero. As a consequence of $\sigma$-additivity, the union of these complements when $n$ ranges over $\mathbb{N}$ has measure that is bounded by the sum of their measures, namely 0 . So the complement of Path $_{\mathcal{E}}$ has $P_{g_{*}}(q)$-measure 0 .

For the second claim, if $\{q\}$ is measurable, then $P_{g_{*}}(q)\left(\left(\left|Q_{0}\right| \backslash\{q\}\right) \times \prod_{i=1}^{+\infty}\left|Q_{i}\right|\right)$ is equal to 0 by (2). The measure of the complement of $\operatorname{Path}_{\mathcal{E}}(q)=\operatorname{Path}_{\mathcal{E}} \cap$ $\left(\{q\} \times \prod_{i=1}^{+\infty}\left|Q_{i}\right|\right)$ therefore also has $P_{g_{*}}(q)$-measure 0 .

\section{Drawing Measurable Selections at Random}

Before we go to the more complicated case of schedulers, we illustrate our basic technique on random choice of measurable selections of a multifunction. We believe this has independent interest.

A multifunction from a set $A$ to a set $B$ is a map $F$ from $A$ to $\mathbb{P}^{*}(B)$, the non-empty powerset of $B$. We say that $F$ is measurable if and only if its graph $\operatorname{Gr} F=\{(x, y) \mid y \in F(x)\}$ is a measurable subset of $X \times Y$. This is one of the many possible notions of measurability for relations, see [12]. The set $F(x)$ is exactly the vertical cut $(\operatorname{Gr} F)_{\mid x}$, showing that for a measurable multifunction, $F(x)$ is a (non-empty) measurable subset of $B$ (see comment before Lemma 2).

A selection for a multifunction $F$ is a map $f: A \rightarrow B$ such that $f(x) \in F(x)$ for every $x \in A$. Every multifunction has a selection: this is the Axiom of Choice. In measure theory, we would like $f$ to be measurable as well. Theorems guaranteeing the existence of measurable selections for certain multifunctions are called measurable selection theorems. There are many of them (see Wagner [18], or Doberkat [8]), but one should remember that measurable multifunctions do not have measurable selections in general: Blackwell showed that there is a multifunction from $[0,1]$ to Baire space $\mathbb{N}^{\mathbb{N}}$ whose graph is closed (hence measurable) but has no measurable selection [3] (see also Example 5.1.7 of [17]).

Given two measurable spaces $X$ and $Y$, let us write $\langle X \rightarrow Y\rangle$ for the space of all measurable maps from $X$ to $Y$, with the weak $\sigma$-algebra. The latter is by definition the subspace $\sigma$-algebra, induced by the inclusion of $|\langle X \rightarrow Y\rangle|$ into the product space $Y^{|X|}$. In other words, this is the smallest $\sigma$-algebra that makes the maps $\varphi \in\langle X \rightarrow Y\rangle \mapsto \varphi(x)$ measurable, for every $x \in|X|$.

More generally, given a multifunction $F:|X| \rightarrow \mathbb{P}^{*}(|Y|)$, we also consider the subspace $\operatorname{Sel}(F)$ of $\langle X \rightarrow Y\rangle$ of all measurable selections of $F$, with the induced $\sigma$-algebra. (Beware that $\operatorname{Sel}(F)$ need not be a measurable subset of $\langle X \rightarrow Y\rangle$.) We again call the latter the weak $\sigma$-algebra, on this subset. In each case, the weak $\sigma$-algebra is generated by subsets that we write $[x \rightarrow E]$, with $x \in|X|$ and $E \in \Sigma_{Y}$, and defined as those measurable functions, resp. those measurable selections of $F$, that map $x$ into $E$.

Assume now a measurable map $g: X \rightarrow \mathcal{P}(Y)$ such that, for every $x \in|X|$, $g(x)$ is concentrated on $F(x)$. For each $x \in|X|$, pick an element $f(x)$ in $F(x)$ with 
probability $g(x)$. The function $f$ is a selection of $F$, but will not be measurable in general. Can we pick $f$ at random so that $f$ is measurable and $f(x)$ is drawn with probability $g(x)$ ? This is the question we answer in the affirmative here.

The problem looks similar to the construction of Wiener measure, a model of Brownian motion, where we would like to draw a map from $\mathbb{R}$ to some topological space at random, and this map should be continuous [2, Section 37]; or to the construction of Skorokhod's J1 topology, which allows one to make sense of random càdlàg functions. Our solution will be simpler, though: measurability is easier to enforce than continuity or being càdlàg.

One can explain the problem in terms of independence [2, Section 5]. Let us remind the reader that independence is not pairwise independence. Consider for example two independent random bits $b_{1}$ and $b_{2}$, and the random variable $b_{3}=b_{1} \oplus b_{2}$, where $\oplus$ is exclusive-or. These random variables are pairwise independent, meaning that any pair among them is formed of independent random variables. However, they are not independent, since given the value of any two, one obtains the third one in a deterministic way. In our case, if we are to draw a measurable map $f$ at random, then the random infinite tuple $(f(x))_{x \in|X|}$ cannot be a collection of independent random variables. However, the results below essentially say that we can choose $f$ measurable at random, in such a way that all countable sequences $\left(f\left(x_{n}\right)\right)_{n \in \mathbb{N}}$ are independent.

A general way to draw several values at random, independently, is to draw them with respect to a product measure. The following says that product measures exists not only for finite products but also for countable products of probability measures. This is well-known, and can even be extended to uncountable products: this is the Łomnick-Ulam Theorem [13, Corollary 5.18].

Lemma 3. Let $\mu_{n}$ be probability measures on the measurable spaces $X_{n}, n \in \mathbb{N}$. There is a unique probability measure $\mu$, written $\bigotimes_{n \in \mathbb{N}} \mu_{n}$, on $\prod_{n \in \mathbb{N}} X_{n}$ such that $\mu\left(\bigcap_{i \in I} \pi_{i}^{-1}\left(E_{i}\right)\right)=\prod_{i \in I} \mu_{i}\left(E_{i}\right)$ for every finite subset $I$ of $\mathbb{N}$, and all measurable subsets $E_{i}$ of $X_{i}, i \in I$.

Proof. Apply Ionescu-Tulcea's Theorem 2 to $Q_{0}=\{*\}, Q_{n+1}=X_{n}$, let $g_{n}$ be the constant map $g_{n}\left(q_{0} q_{1} \cdots q_{n-1}\right)=\mu_{n}$, and note that for every finite set $I$, $\bigcap_{i \in I} \pi_{i}^{-1}\left(E_{i}\right)$ is just the product $\prod_{i=0}^{n} E_{i} \times \prod_{i=n+1}^{+\infty}\left|Q_{i}\right|$, for some $n$ large enough, and where we extend the notation $E_{i}$ to denote $\left|Q_{i}\right|$ for $i \notin I$.

We shall use the following general technique to construct measurable maps.

Lemma 4 (Patching). Let $X, Y$ be measurable spaces, $\left(E_{i}\right)_{i \in I}$ be a countable partition of $|X|$ in measurable subsets $(I \subseteq \mathbb{N})$, and $\left(f_{i}\right)_{i \in I}$ be a matching family of measurable maps from $E_{i}$ to $Y$. The patch $f: X \rightarrow Y$, defined as mapping every $x \in E_{i}$ to $f_{i}(x)$, is a measurable map.

Proof. Categorically, this follows from the fact that $X$ is the coproduct $\sum_{i \in I} E_{i}$. Alternatively, $f^{-1}(E)=\bigcup_{i \in I}\left(f_{i}^{-1}(E) \cap E_{i}\right)$ is measurable as soon as $E$ is.

As an application, we show that a measurable multifunction $F$ that has a measurable selection must have plenty of measurable selections. Precisely, we can fix their values, arbitrarily, at countably many arguments: 
Lemma 5. Let $X, Y$ be measurable spaces, $F: X \rightarrow \mathbb{P}^{*}(Y)$ be a measurable multifunction with a measurable selection $f,\left(x_{i}\right)_{i \in I}$ be countably many points in $X(I \subseteq \mathbb{N})$, and $y_{i}$ be an element of $F\left(x_{i}\right)$ for every $i \in I$. Write $\overrightarrow{\mathbf{y}}$ for $\left(y_{i}\right)_{i \in I}$.

There is a measurable selection $f_{\overrightarrow{\mathbf{y}}}$ of $F$ such that $f_{\overrightarrow{\mathbf{y}}}\left(x_{i}\right)=y_{i}$ for every $i \in I$. Moreover, we can choose $f_{\overrightarrow{\mathbf{y}}}$ in such a way that the mapping $\overrightarrow{\mathbf{y}} \mapsto f_{\overrightarrow{\mathbf{y}}}$ is itself a measurable map from $\prod_{i \in I} F\left(x_{i}\right)$ to $\operatorname{Sel}(F)$.

Proof. Without loss of generality, assume that $I$ is $\mathbb{N}$, or an initial segment $\{0,1, \ldots, n-1\}$ of $\mathbb{N}$. Similarly to vertical cuts, we may define horizontal cuts of $\operatorname{Gr}(F)$ at $y$, namely $\{x \in X \mid y \in F(x)\}$, and they are measurable as well. Write $F^{-1}(y)$ for such a vertical cut. Define $E_{i}$, for each $i \in I$, as $F^{-1}\left(y_{i}\right)>$ $\bigcup_{j=0}^{i-1} F^{-1}\left(y_{j}\right)$. Together with $E_{\infty}=|X| \backslash \bigcup_{i \in I} E_{i}$, they form a partition of $|X|$ in measurable subsets. Define $f_{i}$ as the constant map on $E_{i}$ equal to $y_{i}$ for $i \in I$, and $f_{\infty}$ as the restriction of $f$ to $E_{\infty}$, then form their patch $f_{\overrightarrow{\mathbf{y}}}$, using Lemma 4 . It is plain to see that $f_{\vec{y}}$ is a selection of $F$, and $f_{\vec{y}}$ is measurable.

To show that $\overrightarrow{\mathbf{y}} \mapsto f_{\overrightarrow{\mathbf{y}}}$ is itself measurable, we must show that the set $A$ of tuples $\overrightarrow{\mathbf{y}}$ such that $f_{\overrightarrow{\mathbf{y}}} \in[x \rightarrow E]$ is measurable, for $x \in|X|$ and $E \in \Sigma_{Y}$. For convenience, write $E_{i}(\overrightarrow{\mathbf{y}})$ for the set we called $E_{i}$ above, and similarly with $E_{\infty}$. Let $E_{i}^{\prime}$ be the set of tuples $\overrightarrow{\mathbf{y}} \in \prod_{i \in I} F\left(x_{i}\right)$ such that $y_{i} \in F(x)$ and $y_{j} \notin F(x)$ for every $j, 0 \leq j<i$. $E_{i}^{\prime}$ is measurable since $F(x)$ is measurable: $E_{i}^{\prime}$ is just the rectangle $\prod_{j=0}^{i-1}\left(F\left(x_{j}\right) \backslash F(x)\right) \times\left(F\left(x_{i}\right) \cap F(x)\right) \times \prod_{j \in I, j>i} F\left(x_{j}\right)$. Also, $\overrightarrow{\mathbf{y}} \in E_{i}^{\prime}$ if and only if $x \in E_{i}(\overrightarrow{\mathbf{y}})$. Write $\pi_{i}$ for $i$ th projection. Since $f_{\overrightarrow{\mathbf{y}}}(x) \in E$ if and only if there is an $i \in I$ such that $x \in E_{i}(\overrightarrow{\mathbf{y}})$ and $y_{i} \in E$, or for every $i \in I$, $x \notin E_{i}(\overrightarrow{\mathbf{y}})$ and $f(x) \in E$, it follows that $A=\left(\bigcup_{i \in I} E_{i}^{\prime} \cap \pi_{i}^{-1}(E)\right)$ if $f(x) \notin E$, and $A=\left(\bigcup_{i \in I} E_{i}^{\prime} \cap \pi_{i}^{-1}(E)\right) \cup\left(\bigcap_{i \in I} \overline{E_{i}^{\prime}}\right)$ otherwise. In any case, $A$ is measurable.

Theorem 3 is the keystone of our construction, and allows us to provide foundations to the notion of a random measurable selection.

Theorem 3. Let $X, Y$ be two measurable spaces, and $F: X \rightarrow \mathbb{P}^{*}(Y)$ be a measurable multifunction with a measurable selection. Let also $g: X \rightarrow \mathcal{P}(Y)$ be a measurable map such that, for every $x \in X, g(x)$ is concentrated on $F(x)$.

There is a unique probability measure $\varpi_{g}$ on the space $\operatorname{Sel}(F)$ of measurable selections of $F$ such that $\varpi_{g}\left(\bigcap_{i=1}^{n}\left[x_{i} \rightarrow E_{i}\right]\right)=\prod_{i=1}^{n} g\left(x_{i}\right)\left(E_{i}\right)$ for every finite collection of pairwise distinct points $\left(x_{i}\right)_{1 \leq i \leq n}$ of $X$ and of measurable subsets $\left(E_{i}\right)_{1 \leq i \leq n}$ of $Y$.

Before we prove it, we note the following consequence. Proposition 1 can also be seen as a partial implementation of the Chatterjee-Doyen-Gimbert-Henzinger idea of the introduction in the Markovian case: given a (Markovian) randomized scheduler $g$, draw (Markovian) pure schedulers $f$ at random so that $f(x)$ is drawn with probability $g(x)$.

Proposition 1. Under the assumptions of Theorem 3, let $h: Y \rightarrow \mathbb{R}^{+}$be a measurable map, and $x$ be a point of $X$, then:

$$
\int_{f \in \operatorname{Sel}(F)} h(f(x)) d \varpi_{g}=\int_{y \in Y} h(y) d g(x) .
$$


Proof. Let $\alpha_{x}$ be the measurable map $f \in \operatorname{Sel}(F) \mapsto f(x)$. By the change of variables formula (1), $\int_{f \in \operatorname{Sel}(F)} h(f(x)) d \varpi_{g}=\int_{y \in Y} h(y) d \alpha_{x}\left[\varpi_{g}\right]$. Now note that $\alpha_{x}\left[\varpi_{g}\right](E)=\varpi_{g}\left(\alpha_{x}^{-1}(E)\right)=\varpi_{g}([x \rightarrow E])=g(x)(E)$, so $\alpha_{x}\left[\varpi_{g}\right]=g(x)$.

Proof (of Theorem 3). We use Carathéodory's measure extension Theorem 1. Let $\mathcal{A}$ be the semiring of subsets of the form $\bigcap_{i=1}^{n}\left[x_{i} \rightarrow E_{i}\right]$ given in the statement of the Lemma. To check that this is a semiring, consider any two sets $A$ and $B$ of this form. We must show that $A \backslash B$ is a finite union of elements of $\mathcal{A}$. We may write $A$ as $\bigcap_{i=1}^{n}\left[x_{i} \rightarrow E_{i}\right], B$ as $\bigcap_{j=1}^{m}\left[x_{j}^{\prime} \rightarrow E_{i}^{\prime}\right]$. Then $A \backslash B$ is the finite union of the sets $A_{j}, 1 \leq j \leq m$, defined by: if $x_{j}^{\prime}=x_{k}$ for some (unique) $k, 1 \leq k \leq n$, then $A_{j}=\bigcap_{\substack{i \neq 1 \\ i \neq j}}^{n}\left[x_{i} \rightarrow E_{i}\right] \cap\left[x_{k} \rightarrow E_{i} \backslash E_{j}^{\prime}\right]$, else $A_{j}=\bigcap_{i=1}^{n}\left[x_{i} \rightarrow E_{i}\right] \cap\left[x_{j}^{\prime} \rightarrow \Omega \backslash E_{j}^{\prime}\right]$.

Note that $\mathcal{A}$ generates the weak $\sigma$-algebra on $\operatorname{Sel}(F)$, by definition. Assume there is a map $\mu: \mathcal{A} \rightarrow[0,+\infty]$ that satisfies the formula given in the statement of the Lemma: $\mu\left(\bigcap_{i=1}^{n}\left[x_{i} \rightarrow E_{i}\right]\right)=\prod_{i=1}^{n} g\left(x_{i}\right)\left(E_{i}\right)$. For now, this is an assumption, not a definition. For it to be a definition, we would need to check that this is unambiguous: if $\bigcap_{i=1}^{n}\left[x_{i} \rightarrow E_{i}\right]=\bigcap_{j=1}^{m}\left[x_{j}^{\prime} \rightarrow E_{j}^{\prime}\right]$, we should verify that $\prod_{i=1}^{n} g\left(x_{i}\right)\left(E_{i}\right)=\prod_{j=1}^{m}\left[x_{j}^{\prime} \rightarrow E_{j}^{\prime}\right]$. This will be easier to prove later. Until then, we concentrate on the more interesting question of $\sigma$-additivity.

Let $A_{k}=\bigcap_{i \in I_{k}}\left[x_{k i} \rightarrow E_{k i}\right], k \in \mathbb{N}$, be a countable family of disjoint elements of $\mathcal{A}$ whose union is some element $A$ of $\mathcal{A}$ again, where each index set $I_{k}$ is finite. We must show that $\mu(A)=\sum_{k=0}^{+\infty} \mu\left(A_{k}\right)$.

There is a simple trick to prove this: we exhibit a measure $\left(s_{J}\left[\mu_{J}\right]\right.$ below) that coincides with $\mu$ on $A$ and each $A_{k}, k \in \mathbb{N}$. We shall call this the Eomnick-Ulam trick, since this also subtends the classical proof of the Eomnick-Ulam theorem.

Observe that the set of points $\left(x_{k i}\right)_{k \in \mathbb{N}, i \in I_{k}}$ is countable. For each countable set $J$ of points of $X$, let $s_{J}: \prod_{x \in J} F(x) \rightarrow \operatorname{Sel}(F)$ be the map $\overrightarrow{\mathbf{y}} \mapsto f_{\overrightarrow{\mathbf{y}}}$ given in Lemma 5 . Let $\mu_{J}$ be the product probability measure $\bigotimes_{x \in J} g(x)$ on $\prod_{x \in J} F(x)$, as given in Lemma 3 .

By definition, $s_{J}\left[\mu_{J}\right](\mathcal{E})=\mu_{J}\left(s_{J}^{-1}(\mathcal{E})\right)$ for every measurable subset $\mathcal{E}$ of $\operatorname{Sel}(F)$. In particular, if $J$ contains all the points $x_{1}, \ldots, x_{n}$, then $s_{J}\left[\mu_{J}\right]\left(\bigcap_{i=1}^{n}\left[x_{i}\right.\right.$ $\left.\left.\rightarrow E_{i}\right]\right)=\mu_{J}\left\{\overrightarrow{\mathbf{y}} \in \prod_{x \in J} F(x) \mid \forall i, 1 \leq i \leq n \cdot y_{x_{i}} \in E_{i}\right\}\left(\operatorname{since} s_{J}(\overrightarrow{\mathbf{y}})\left(x_{i}\right)=\right.$ $f_{\overrightarrow{\mathbf{y}}}\left(x_{i}\right)=y_{x_{i}}$, where we agree to write the tuple $\overrightarrow{\mathbf{y}}$ with indices in $J$, namely, as $\left.\left(y_{x}\right)_{x \in J}\right)=\mu_{J}\left(\bigcap_{i=1}^{n} \pi_{x_{i}}^{-1}\left(E_{i}\right)\right)$. By definition of $\mu_{J}$, this is equal to $\prod_{i=1} g\left(x_{i}\right)\left(E_{i}\right)$, hence to $\mu\left(\bigcap_{i=1}^{n}\left[x_{i} \rightarrow E_{i}\right]\right)$.

In other words, $s_{J}\left[\mu_{J}\right]$ coincides with $\mu$ on all subsets of $\mathcal{A}$ of the form $\bigcap_{i=1}^{n}\left[x_{i} \rightarrow E_{i}\right]$ where every $x_{i}$ is in $J$. It is certainly not the case in general that $s_{J}\left[\mu_{J}\right]$ and $\mu$ coincide! The condition that every $x_{i}$ is in $J$ is crucial. This condition is satisfied by $A$ and every $A_{k}, k \in \mathbb{N}$, provided we take $J=\left(x_{k i}\right)_{k \in \mathbb{N}, i \in I_{k}}$. Since $s_{J}\left[\mu_{J}\right]$ is $\sigma$-additive, the equation $\mu(A)=\sum_{k=0}^{+\infty} \mu\left(A_{k}\right)$ holds.

This construction also shows that $\mu$ indeed exists, something we had deferred the verification of. The problem was to show that if $\bigcap_{i=1}^{n}\left[x_{i} \rightarrow E_{i}\right]=$ $\bigcap_{j=1}^{m}\left[x_{j}^{\prime} \rightarrow E_{j}^{\prime}\right]$ then $\prod_{i=1}^{n} g\left(x_{i}\right)\left(E_{i}\right)=\prod_{j=1}^{m}\left[x_{j}^{\prime} \rightarrow E_{j}^{\prime}\right]$. Take $J=\left\{x_{1}, \cdots, x_{n}\right\} \cup$ $\left\{x_{1}^{\prime}, \cdots, x_{m}^{\prime}\right\}$. The inverse image by $s_{J}$ of the set $\bigcap_{i=1}^{n}\left[x_{i} \rightarrow E_{i}\right]=\bigcap_{j=1}^{m}\left[x_{j}^{\prime} \rightarrow\right.$ 
$\left.E_{j}^{\prime}\right]$ is equal to $\bigcap_{i=1}^{n} \pi_{x_{i}}^{-1}\left(E_{i}\right)=\bigcap_{j=1}^{m} \pi_{x_{j}^{\prime}}^{-1}\left(E_{j}^{\prime}\right)$, and its $\mu_{J}$-measure is $\prod_{i=1}^{n} g\left(x_{i}\right)$ $\left(E_{i}\right)=\prod_{j=1}^{m}\left[x_{j}^{\prime} \rightarrow E_{j}^{\prime}\right]$, by definition of the product probability measure.

The existence of $\varpi_{g}$ follows directly from Carathéodory's theorem. It is clear that it is a probability measure. Uniqueness follows from the fact that probability measures are uniquely determined by their values on any $\pi$-system that generates the $\sigma$-algebra [2, Theorem 3.3]. A $\pi$-system is a collection of sets that is closed under binary intersections, and certainly $\mathcal{A}$ qualifies, as a semiring.

\section{IT Automata}

Consider the following simple form of non-deterministic automaton, which we call an IT automaton (for Ionescu-Tulcea automaton): a tuple $(Z, \Lambda, F)$ where $Z$, $\Lambda$ are measurable spaces, and $F: Z \Lambda^{*} \rightarrow \mathbb{P}^{*}(\Lambda)$ is a measurable multifunction with a measurable selection. $Z$ can be thought as a space of input values, $\Lambda$ as a space of states, and $F$ as a generalized transition relation, which given a finite history $z \lambda_{1} \cdots \lambda_{n}$ produces a non-empty set $F\left(z \lambda_{1} \cdots \lambda_{n}\right)$ of possible next states. The idea is that the system starts with some input value $z$, goes to the first state $\lambda_{1} \in F(z)$, then to a second state $\lambda_{2} \in F\left(z \lambda_{1}\right), \ldots$, to an $n$th state $\lambda_{n} \in F\left(z \lambda_{1} \cdots \lambda_{n-1}\right)$, and so on. In other words, IT automata are just like non-deterministic automata, except on possibly infinite state spaces and with a history-dependent transition relation.

We use the notation $\Lambda^{*}$ for the space of all finite words on the alphabet $\Lambda$, which we equate with the countable coproduct $\sum_{n \in \mathbb{N}} \Lambda^{n}$. We also write $Z \Lambda^{*}$ instead of $Z \times \Lambda^{*}$, and will more generally drop the $\times$ symbol in cases where this is not ambiguous. Accordingly, we write $z \lambda_{1} \cdots \lambda_{n}$ in word notation, instead of as the tuple $\left(z, \lambda_{1}, \cdots, \lambda_{n}\right)$. We have already done so before.

Since an IT automaton starting from input value $z$ will produce infinitely many states $\lambda_{1}, \ldots, \lambda_{n}$, it is natural to study the space $Z \Lambda^{\mathbb{N}}$ of infinite paths of the automaton, where $\Lambda^{\mathbb{N}}$ is the product of countably infinitely many copies of $\Lambda$. (This is written $\Lambda^{\omega}$ in language theory.) The $\sigma$-algebra on $Z \Lambda^{\mathbb{N}}$ is generated by so-called cylinders, which are exactly the products $X_{0} \Lambda_{1} \Lambda_{2} \ldots \Lambda_{n} \Lambda^{\mathbb{N}}$ with $X_{0} \in \Sigma_{Z}$ and $\Lambda_{i} \in \Sigma_{\Lambda}, 1 \leq i \leq n$, and $n \in \mathbb{N}$.

A randomized scheduler for such an IT automaton is a measurable map $g: Z \Lambda^{*} \rightarrow \mathcal{P}(\Lambda)$ such that $g\left(z \lambda_{1} \cdots \lambda_{n}\right)$ is concentrated on $F\left(z \lambda_{1} \cdots \lambda_{n}\right)$ for every $z \lambda_{1} \cdots \lambda_{n} \in Z \Lambda^{*}$.

Given a measurable subset $\mathcal{E}$ of $Z \Lambda^{\mathbb{N}}$, and an input value $z \in|Z|$, the probability that the induced infinite path lies in $\mathcal{E}$, where $\lambda_{n}$ is chosen at random with probability $g\left(z \lambda_{1} \cdots \lambda_{n-1}\right)$ at each step, is given by Theorem 2 , with $Q_{0}=Z \Lambda^{*}$, $Q_{i}=\Lambda$ for every $i \geq 1$, and letting $g_{n}$ be the restriction of $g$ to $Z \Lambda^{n-1}$. Explicitly:

Proposition 2. Let $Z, \Lambda$ be two measurable spaces, and $g: Z \Lambda^{*} \rightarrow \mathcal{P}(\Lambda)$ be a measurable map. There is a unique map $P_{g}: Z \Lambda^{*} \rightarrow \mathcal{P}\left(Z \Lambda^{\mathbb{N}}\right)$ such that $P_{g}(w)\left(X_{0}\right.$ $\Lambda_{1} \Lambda_{2} \cdots \Lambda_{n} \cdots \Lambda_{n+m} \Lambda^{\mathbb{N}}$ ) is equal to:

$$
\chi_{X_{0}}(z) \chi_{\Lambda_{1}}\left(\lambda_{1}\right) \cdots \chi_{\Lambda_{n}}\left(\lambda_{n}\right) \int_{\lambda_{n+1} \in \Lambda_{n+1}} d g(w) \int_{\lambda_{n+2} \in \Lambda_{n+2}} d g\left(w \lambda_{n+1}\right) \cdots \int_{\lambda_{n+m} \in \Lambda_{n+m}} d g\left(w \lambda_{n+1} \cdots \lambda_{n+m-1}\right)
$$


for all measurable subsets $X_{0}$ of $Z, \Lambda_{i}$ of $\Lambda(1 \leq i \leq m, n \leq m)$, and elements $w=z \lambda_{1} \cdots \lambda_{n} \in Z \Lambda^{*}$. Moreover, $P_{g}$ is measurable.

A pure scheduler for the IT automaton $(Z, \Lambda, F)$ is just a measurable selection of $F$ : given the history $z \lambda_{1} \cdots \lambda_{n-1}$, pick a next state $\lambda_{n}$ from $F\left(z \lambda_{1} \cdots \lambda_{n-1}\right)$.

Given a pure scheduler $f$, define $\widetilde{f}: Z \Lambda^{*} \rightarrow Z \Lambda^{\mathbb{N}}$ so that $\widetilde{f}\left(z \lambda_{1} \cdots \lambda_{n}\right)$ is the unique infinite path that we obtain by starting with the history $z \lambda_{1} \cdots \lambda_{n}$ and repeatedly computing next states, using $f: \widetilde{f}\left(z \lambda_{1} \cdots \lambda_{n}\right)=z \lambda_{1} \cdots \lambda_{n} \lambda_{n+1} \cdots \lambda_{n+m} \cdots$ where $\lambda_{n+k+1}=f\left(z \lambda_{1} \cdots \lambda_{n} \lambda_{n+1} \cdots \lambda_{n+k}\right)$, for every $k \in \mathbb{N}$.

One might think of doing the following. Fix an IT automaton $(Z, \Lambda, F)$, and a randomized scheduler $g$ for this automaton. Pick a pure scheduler $f$ at random, with respect to the probability measure $\varpi_{g}$ given by Theorem 3 , and show that the probability that $\widetilde{f}(z)$ falls into any given measurable set $\mathcal{E}$ of infinite paths is equal to the probability $P_{g}(z)(\mathcal{E})$ given in Proposition 2. We did the computation, and checked that this indeed holds... except this is all wrong! This does not make sense unless the map $f \mapsto \widetilde{f}(z)$ (for $z \in|Z|$ fixed) is measurable. We cannot dismiss the problem: this is the central question here.

To state it another way, the weak $\sigma$-algebra on the space of pure schedulers has to be replaced by a larger one: the $\widetilde{\text { weak }} \sigma$-algebra on the set $|\operatorname{Sel}(F)|$ of pure schedulers is the smallest that makes every map $f \in|\operatorname{Sel}(F)| \mapsto \widetilde{f}\left(z \lambda_{1} \cdots \lambda_{n}\right)$ measurable, for every $z \lambda_{1} \cdots \lambda_{n} \in Z \Lambda^{*}$. We write $\widetilde{\operatorname{Sel}}(F)$ for $|\operatorname{Sel}(F)|$ with the $\widetilde{\text { weak }} \sigma$-algebra.

For $w=z \lambda_{1} \cdots \lambda_{n} \in Z \Lambda^{*}$, and a measurable subset $\mathcal{E}$ of $\Lambda^{\mathbb{N}}$, let us write $w \mathcal{E}$ for $\{z\} \times\left\{\lambda_{1}\right\} \times \cdots \times\left\{\lambda_{n}\right\} \times \mathcal{E}$. The weak $\sigma$-algebra is generated by the sets $[w \stackrel{\sim}{\rightarrow} w]$, defined as the set of pure schedulers $f$ such that $\widetilde{f}(w) \in w \mathcal{E}$.

We wish to define our probability measure $\widetilde{\omega}_{g}$ on pure schedulers $f$ by saying that the probability that $\widetilde{f}(w) \in w \mathcal{E}$ (for any fixed $w, \mathcal{E}$ ) is exactly $P_{g}(w)(\mathcal{E})$, where $P_{g}$ is given in Proposition 2: namely, $\widetilde{\omega}_{g}([w \widetilde{\rightarrow} w \mathcal{E}])=P_{g}(w)(\mathcal{E})$. That cannot be enough to define $\widetilde{\omega}_{g}$, and we need to at least define $\widetilde{\omega}_{g}\left(\bigcap_{i=1}^{n}\left[w_{i} \widetilde{\rightarrow}\right.\right.$ $\left.\left.w_{i} \mathcal{E}_{i}\right]\right)$ for all finite intersections of sets $\left[w_{i} \widetilde{\rightarrow} w_{i} \mathcal{E}_{i}\right]$. Now there is a big difference with the case of random measurable selections (Section 3): the choices we make for $\widetilde{f}\left(w_{i}\right)$ for different indices $i$ cannot in general be independent. Indeed, imagine we have chosen $\widetilde{f}\left(w_{i}\right)$, for some $i$, to be $w_{i} \lambda_{n+1} \cdots \lambda_{n+m} \cdots$ : then we have no choice for $\widetilde{f}\left(w_{i} \lambda_{n+1}\right)$, and also for $\widetilde{f}\left(w_{i} \lambda_{n+1} \lambda_{n+2}\right), \ldots$, which must all be equal to $w_{i} \lambda_{n+1} \cdots \lambda_{n+m} \cdots$. This is the consistency condition mentioned in the proof of Proposition 3 below: in general, if we have chosen $\widetilde{f}\left(w_{i}\right)$ as $w_{i} \omega_{i}$ for some $\omega_{i} \in \Lambda^{\mathbb{N}}$, and later we need to choose $\widetilde{f}\left(w_{j}\right)$ where $w_{i}$ is a prefix of $w_{j}$, and $w_{j}$ is a prefix of $w_{i} \omega_{i}$, then we must choose it as $\widetilde{f}\left(w_{j}\right)=w_{i} \omega_{i}$.

We still proceed in a manner similar to Section 3 . We now need an extra assumption: say that measurable space $X$ has measurable diagonal if and only if the diagonal $\Delta=\operatorname{Gr}(=)=\{(x, x)|x \in| X \mid\}$ is measurable in $X \times X$. Dravecký [9, Theorem 1] shows a number of equivalent conditions. One of these is that $X$ has measurable diagonal if and only if there is a countable family $\left(E_{n}\right)_{n \in \mathbb{N}}$ of measurable subsets of $X$ that separates points, that is, such that for any two 
distinct points $x, y$, there is an $E_{n}$ that contains one and not the other. This is true for all Polish spaces, notably. Another one is that $X$ has measurable diagonal if and only if for every measurable function $f$ from an arbitrary measurable space $Y$ to $X$, the obvious multifunction $y \in Y \mapsto\{f(y)\}$ is measurable. This apparent tautology is wrong when $X$ does not have measurable diagonal! The canonical counter-example is $Y=X, f=\mathrm{id}_{X}$ : the corresponding multifunction is precisely the one whose graph is $\Delta$. Finally, every one-element subset $\{x\}$ of a space $X$ with measurable diagonal is measurable in $X$; indeed, $\{x\}$ is the vertical cut $\Delta_{\mid x}$.

Proposition 3. Let $(Z, \Lambda, F)$ be an IT automaton, with a randomized scheduler $g: Z \Lambda^{*} \rightarrow \mathcal{P}(\Lambda)$. Assume that $Z$ and $\Lambda$ both have measurable diagonals. Let $P_{g}: Z \Lambda^{*} \rightarrow \mathcal{P}\left(Z \Lambda^{\mathbb{N}}\right)$ be the probability-on-paths map given in Proposition 2. There is a probability measure $\widetilde{\omega}_{g}$ on $\widetilde{\operatorname{Sel}}(F)$ such that $\widetilde{\omega}_{g}([z \widetilde{\mathcal{E}}])=P_{g}(z)(\mathcal{E})$ for all $z \in Z$ and $\mathcal{E} \in \Sigma_{Z \Lambda^{\mathbb{N}}}$.

Proof. (Outline. The technical details are relegated to Appendix A.) As a notational help, we write $w$, possibly subscripted or primed, for finite words in $Z \Lambda^{*}$, and $\omega$, possibly subscripted or primed, for infinite words in $\Lambda^{\mathbb{N}}$.

Let us write $\preceq$ for the prefix relation on finite and infinite words. Say that a set of words $A$ of $Z \Lambda^{*}$ is prefix-closed if and only if for every $w \in Z \Lambda^{*}$ that is a prefix of some element of $A, w$ is in $A$ as well.

Let $\mathcal{A}$ be the semiring of all finite intersections of basic measurable subsets $\bigcap_{i=1}^{n}\left[w_{i} \widetilde{\rightarrow} w_{i} \mathcal{E}_{i}\right]$. By adding extra words if needed, we may assume that $\mathcal{I}=$ $\left\{w_{1}, \ldots, w_{n}\right\}$ is prefix-closed.

It is hard to even attempt to describe explicitly the values of $\widetilde{\omega}_{g}$ on elements of $\mathcal{A}$. In the proof of Theorem 3, we had eventually shown that the value of the desired measure $\varpi_{g}$ coincided with the image measure of some other measure defined on a product space for sufficiently small parts of the semiring. We define $\widetilde{\omega}_{g}$ on $\mathcal{A}(\mathcal{I})$ by a similar Eomnick-Ulam-like trick: through image measures of some measures $\mu_{\mathcal{W}}$ under maps $\alpha_{\mathcal{W}}$, for countably infinite subsets $\mathcal{W}$ of $Z \Lambda^{*}$.

Given a countably infinite set $\mathcal{W}=\left(w_{j}\right)_{j \in J}$ of words in $Z \Lambda^{*}$ (with $J=$ $\mathbb{N} \backslash\{0\}$, say), let $C s t_{\mathcal{W}}$ be the subspace of $\prod_{w \in \mathcal{W}} \operatorname{Path}_{F}(w)$ consisting of those tuples $\left(w_{j} \omega_{j}\right)_{j \in J}$ that are consistent: for all $i, j \in J$ such that $w_{i} \preceq w_{j} \preceq w_{i} \omega_{i}$, then $w_{i} \omega_{i}=w_{j} \omega_{j}$. In pictures, if the leftmost two zones are equal then the rightmost zones are equal, too:

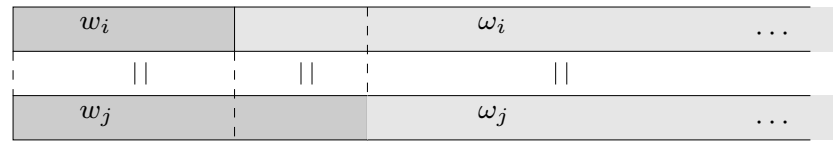

Using Ionescu-Tulcea's Theorem 2, we build a probability measure $\mu_{\mathcal{W}}$ on $C s t_{\mathcal{W}}$ (Proposition 5 in Appendix A). Intuitively, $\mu_{\mathcal{W}}$ picks $w_{1} \omega_{1}$ at random using probability measure $P_{g}\left(w_{1}\right)$, then picks $w_{2} \omega_{2}, \ldots, w_{j} \omega_{j}, \ldots$, as follows. At step $j$, if $w_{i} \preceq w_{j} \preceq w_{i} \omega_{i}$ for some previous $i, 1 \leq i<j$, then we pick $w_{j} \omega_{j}$, deterministically, as equal to $w_{i} \omega_{i}$, enforcing consistency; otherwise, we pick $w_{j} \omega_{j}$ at random using probability measure $P_{g}\left(w_{j}\right)$. All this makes sense provided 
we sort $\mathcal{W}$ topologically, i.e., we choose the indexing scheme so that any $w_{i}$ that is a prefix of $w_{j}$ occurs before $w_{j}$, viz., $i \leq j$ (Lemma 6 in Appendix A).

Given $w \in \mathcal{W}$, say $w=w_{j}$, let us write $[w: \mathcal{E}]$ for the set of consistent tuples $\overrightarrow{\mathbf{w} \omega}$ in $C s t_{\mathcal{W}}$ such that $\omega_{j} \in \mathcal{E}$. One can show that, given any finite prefix-closed set $\left\{w_{1}, \ldots, w_{n}\right\}$ of words in $Z \Lambda^{*}$, the value $\mu_{\mathcal{W}}\left(\bigcap_{i=1}^{n}\left[w_{i}: \mathcal{E}_{i}\right]\right)$ is the same for all countably infinite subsets $\mathcal{W}$ that contain $w_{1}, \ldots, w_{n}$ (Lemma 9 in Appendix A). The formula we obtain simplifies when $n=1$ and $w_{1}$ is a single letter $z \in Z$ : $\mu_{\mathcal{W}}([z: \mathcal{E}])=P_{g}(z)(z \mathcal{E})($ Lemma 10 in Appendix A).

We take the image measure of $\mu_{\mathcal{W}}$ under a measurable map $\alpha_{\mathcal{W}}: \overrightarrow{\mathbf{w} \omega} \in$ $C_{s t} t_{\mathcal{W}} \mapsto f_{\overrightarrow{\mathrm{w} \omega}} \in \widetilde{\operatorname{Sel}}(F)$ that retrieves a canonical pure scheduler from a consistent set of tuples (Proposition 6 in Appendix A), defined in such a way that $\widetilde{f}_{\overrightarrow{\mathrm{w} \omega}}\left(w_{j}\right)=w_{j} \omega_{j}$ for every $j \in J$. This is done by patching, similarly to Lemma 5 .

Note that $\alpha_{\mathcal{W}}\left[\mu_{\mathcal{W}}\right]\left(\bigcap_{i=1}^{n}\left[w_{i} \widetilde{\rightarrow} w_{i} \mathcal{E}_{i}\right]\right)$ is independent of $\mathcal{W}$, provided that $\mathcal{W}$ contains the prefix-closed subset $\left\{w_{1}, \ldots, w_{n}\right\}$. Indeed, $\alpha_{\mathcal{W}}\left[\mu_{\mathcal{W}}\right]\left(\bigcap_{i=1}^{n}\left[w_{i} \widetilde{\rightrightarrows}\right.\right.$ $\left.\left.w_{i} \mathcal{E}_{i}\right]\right)=\mu_{\mathcal{W}}\left(\bigcap_{i=1}^{n}\left[w_{i}: \mathcal{E}_{i}\right]\right)$, which we have shown independent of $\mathcal{W}$. We can therefore define $\widetilde{\omega}_{g}$ as coinciding with $\alpha_{\mathcal{W}}\left[\mu_{\mathcal{W}}\right]$ on those elements $\bigcap_{i=1}^{n}\left[w_{i} \widetilde{\rightrightarrows}\right.$ $\left.w_{i} \mathcal{E}_{i}\right]$ of $\mathcal{A}$ with $\left\{w_{1}, \ldots, w_{n}\right\} \subseteq \mathcal{W}$. As such, it is $\sigma$-additive on $\mathcal{A}$ : as in the proof of Theorem 3 , let $A_{k}=\bigcap_{w \in \mathcal{I}_{k}}\left[w \widetilde{\rightarrow} \mathcal{E}_{w i}\right], k \in \mathbb{N}$, be a countable family of disjoint elements of $\mathcal{A}$, where $\mathcal{I}_{k}$ is finite and prefix-closed, and $A=\bigcap_{w \in \mathcal{I}}[w \widetilde{\rightarrow}$ $\left.\mathcal{E}_{w}\right]$ be their union, assumed in $\mathcal{A}$, with $\mathcal{I}$ prefix-closed again. Then $\widetilde{\omega}_{g}(A)=$ $\sum_{k \in \mathbb{N}} \widetilde{\omega}_{g}\left(A_{k}\right)$, since $\widetilde{\omega}_{g}$ coincides with the measure $\alpha_{\mathcal{W}}\left[\mu_{\mathcal{W}}\right]$ on $A$ and every $A_{k}$, for $\mathcal{W}=\bigcup_{k \in \mathbb{N}} \mathcal{I}_{k} \cup \mathcal{I}$ (which is countable).

Finally, $\widetilde{\omega}_{g}([z \widetilde{\rightarrow} z \mathcal{E}])=\mu_{\mathcal{W}}([z: \mathcal{E}])=P_{g}(z)(z \mathcal{E})$, for every measurable subset $\mathcal{E}$ of $\Lambda^{\mathbb{N}}$. Since $P_{g}(z)$ is concentrated on $\operatorname{Path}_{F}(z)$, hence on $z \Lambda^{\mathbb{N}}$, for every measurable subset $\mathcal{E}$ of $Z \Lambda^{\mathbb{N}}, \widetilde{\omega}_{g}([z \widetilde{\mathcal{G}}])=\widetilde{\omega}_{g}\left(\left[z \widetilde{\rightrightarrows} z \mathcal{E}_{\mid z}\right]\right)=P_{g}(z)\left(z \mathcal{E}_{\mid z}\right)=$ $P_{g}(z)\left(\mathcal{E} \cap z \Lambda^{\mathbb{N}}\right)=P_{g}(z)(\mathcal{E})$.

We can now integrate on infinite paths $\omega$ with respect to $P_{g}(z)$, or on pure schedulers, and this will give the same average value:

Proposition 4. Under the assumptions of Proposition 3, let $h: Z \Lambda^{\mathbb{N}} \rightarrow \mathbb{R}^{+}$be a measurable map, and $z \in Z$, then:

$$
\int_{f \in \widetilde{\operatorname{Sel}}(F)} h(\widetilde{f}(z)) d \widetilde{\omega}_{g}=\int_{\omega \in Z \Lambda^{\mathbb{N}}} h(\omega) d P_{g}(z) .
$$

Proof. Let $\widetilde{\alpha}_{z}: f \mapsto \widetilde{f}(z)$. This is a measurable map, since $\widetilde{\alpha}_{z}^{-1}(\mathcal{E})=[z \widetilde{\mathcal{E}}]$. The left-hand side is $\int_{f \in \widehat{\operatorname{Sel}}(F)} h\left(\widetilde{\alpha}_{z}(f)\right) d \widetilde{\omega}_{g}=\int_{\omega \in Z \Lambda^{\mathbb{N}}} h(\omega) d \widetilde{\alpha}_{z}\left[\widetilde{\omega}_{g}\right]$ by the change of variables formula (1). Proposition 3 states precisely that $\widetilde{\alpha}_{z}\left[\widetilde{\omega}_{g}\right]=P_{g}(z)$.

We may think of $h$ as a payoff function on infinite paths. The above shows that the average payoff with respect to $P_{g}(z)$ is also the average of the individual payoffs $h(\widetilde{f}(z))$ one would get by drawing a pure scheduler $f$ at random instead.

Fact 1 then implies that the value of the average payoff is bounded by the payoff evaluated on two pure schedulers $f^{-}$and $f^{+}$: 
Corollary 1 (Randomness for Free). Under the assumptions of Proposition 3, let $h: Z \Lambda^{\mathbb{N}} \rightarrow \mathbb{R}^{+}$be a measurable map, and $z \in Z$. There are two pure schedulers $f^{-}$and $f^{+}$in $\operatorname{Sel}(F)$ such that:

$$
h\left(\widetilde{f^{-}}(z)\right) \leq \int_{\omega \in Z \Lambda^{\mathbb{N}}} h(\omega) d P_{g}(z) \leq h\left(\widetilde{f^{+}}(z)\right) .
$$

\section{Conclusion}

We have established a few "randomness for free" type theorems for measurable multifunctions first, for IT automata second. The results are pleasing, and our assumptions are fairly minimal. Our proofs use fairly simple ideas, too: there is the Chatterjee-Doyen-Gimbert-Henzinger idea of drawing measurable selections/pure schedulers at random first, and this makes sense because of a combination of patching, of Carathéodory's measure extension theorem, and of a Łomnik-Ulam type trick.

Where should we go next? One may push the results on IT automata to partially observable IT automata. Instead of a measurable transition multifunction $F: Z \Lambda^{*} \rightarrow \mathbb{P}^{*}(\Lambda)$, such automata have a measurable transition multifunction $F: Z \Lambda^{*} \rightarrow \mathbb{P}^{*}(\Xi)$, where $\Xi$ is an (additional) measurable space $\Xi$ of actual states. Such states $q$ are mapped to observable states $\lambda \in \Lambda$ by a measurable map $\tau: Z \Lambda^{*} \times \Xi \rightarrow \Lambda$; this may depend on the past history $w \in Z \Lambda^{*}$, viz., $\lambda=\tau(w, q)$. We pick the next observable state $\lambda$ after history $w$ by picking $q$ from $F(w)$, then computing $\tau(w, q)$. Modifying the notion of consistent paths as required, it seems feasible to prove an analogue of Proposition 3 for partially observable IT automata. The $\sigma$-algebra on $\operatorname{Sel}(F)$ needs to be changed again! so that we cannot reuse Proposition 3 as is. Once this is done, we can proceed to stochastic automata [4]. Given a stochastic automaton with state space $Q$ and action space $L$, take $Z=Q, \Lambda=(L \times Q)_{\perp}$ (writing $X_{\perp}$ for $X+\{\perp\}$ ), and $\Xi=\mathcal{P}(\Lambda)$. Taking $g$ to be the second projection map $\pi_{2}$, Theorem 3 allows us to draw the observation maps $\tau: Q(L \times Q)_{\perp}^{*} \times \mathcal{P}\left((L \times Q)_{\perp}\right) \rightarrow(L \times Q)_{\perp}$ at random, and this will simulate the probabilistic choice of $q^{\prime}$ with respect to $\mu$ described in the introduction. Combining this with the alluded "randomness for free" result for partially observable IT automata, we hope that it would settle the "randomness for free" question for general, stochastic automata.

\section{References}

1. Assalé Adjé, Olivier Bouissou, Jean Goubault-Larrecq, Éric Goubault, and Sylvie Putot. Analyzing numerical programs with partially known distributions. In Ernie Cohen and Andrey Rybalchenko, editors, Proc. 5th IFIP TC2 WG 2.3 Conf. Verified Software-Theories, Tools, and Experiments (VSTTE'13), Lecture Notes in Computer Science. Springer Verlag, 2013. http://www.lsv.ens-cachan.fr/ Publis/PAPERS/PDF/ABGGP-vstte13.pdf.

2. Patrick Billingsley. Probability and Measure. Wiley series in probability and mathematical statistics. John Wiley and Sons, 3rd edition, 1995. 
3. David Harold Blackwell. A Borel set not containing a graph. Annals of Mathematical Statistics, 39:1345-1347, 1968.

4. Stefano Cattani, Roberto Segala, Marta Kwiatkowska, and Gethin Norman. Stochastic transition systems for continuous state spaces and non-determinism. In Proc. 8th Intl. Conf. Foundations of Software Science and Computational Structures (FOSSACS'05), pages 125-139. Springer Verlag LNCS 3441, 2005.

5. Krishnendu Chatterjee, Laurent Doyen, Hugo Gimbert, and Thomas A. Henzinger. Randomness for free. In Proc. 35th Intl. Symp. Mathematical Foundations of Computer Science (MFCS'10), pages 246-257. Springer Verlag LNCS 6281, 2010.

6. Josée Desharnais, Abbas Edalat, and Prakash Panangaden. Bisimulation for labelled Markov processes. Information and Computation, 179(2):163-193, 2002.

7. Josée Desharnais, Vineet Gupta, Radha Jagadeesan, and Prakash Panangaden. Metrics for labelled Markov processes. Theoretical Computer Science, 318:323$354,2004$.

8. Ernst-Erich Doberkat. Stochastic Relations: Foundations for Markov Transition Systems. Studies in Informatics Series. Chapman and Hall/CRC, 2007.

9. Jozef Dravecký. Spaces with measurable diagonal. Matematický Casopis, 25(1):39, 1975.

10. Norm Ferns, Prakash Panangaden, and Doina Precup. Bisimulation metrics for continuous Markov decision processes. SIAM Journal on Computing, 40(6):16621714, 2011.

11. Michèle Giry. A categorical approach to probability theory. In Bernhard Banaschewski, editor, Categorical Aspects of Topology and Analysis, pages 68-85. Springer Verlag LNM 915, 1981.

12. Charles J. Himmelberg. Measurable relations. Fundamenta Mathematicae, 87:53$71,1975$.

13. Olav Kallenberg. Foundations of Modern Probability. Probability and its Applications. Springer, 2nd edition edition, 2002.

14. Prakash Panangaden. Probabilistic relations. In C. Baier, M. Huth, M. Kwiatkowska, and M. Ryan, editors, Proceedings of PROBMIV'98, pages 59-74, 1998. http://www.cs.bham.ac.uk/ mzk/probmiv/prelproc98/.

15. Prakash Panangaden. Probability and measure for concurrency theorists. Theoretical Computer Science, 253(2):287-309, February 2001.

16. Prakash Panangaden. Labelled Markov Processes. Imperial College Press, 2009.

17. Sashi Mohan Srivastava. A Course on Borel Sets, volume 180 of Graduate Texts in Mathematics. Springer Verlag, 1998.

18. Daniel H. Wagner. Survey of measurable selection theorems: An update. In Measure Theory, Oberwolfach, 1979, pages 176-219. Springer Verlag LNM 794, 1980.

\section{A Auxiliary Results Needed for Proposition 3}

In the following, we fix an IT automaton $(Z, \Lambda, F)$ with a randomized scheduler $g: Z \Lambda^{*} \rightarrow \mathcal{P}(\Lambda)$. We also assume that $Z$ and $\Lambda$ both have measurable diagonals. Let $P_{g}: Z \Lambda^{*} \rightarrow \mathcal{P}\left(Z \Lambda^{\mathbb{N}}\right)$ be the probability-on-paths map given in Proposition 2 .

In the rest of this section, we fix a countably infinite set $\mathcal{W}$ of words in $Z \Lambda^{*}$. We let $J=\{1,2, \cdots\}=\mathbb{N} \backslash\{0\}$. This will serve as an index set.

Lemma 6. One can write $\mathcal{W}$ as a family $\left(w_{j}\right)_{j \in J}$ in such a way that for all $i, j \in J$, if $w_{i} \preceq w_{j}$ then $i \leq j$. 
In other words, one can sort the words topologically. Proving this involves showing that the order type of $\mathcal{W}$ under the prefix ordering is at most $\omega$.

Proof. There are only countably many elements of $Z$, resp. $\Lambda$, that can occur in any word from $\mathcal{W}$, so one can attribute each of them a unique natural number. Equate each element with the corresponding natural number. Each word $w=z \lambda_{1} \cdots \lambda_{n}$ can now be encoded as $p_{1}^{z} p_{2}^{\lambda_{1}} \cdots p_{n+1}^{\lambda_{n}}$, where $p_{1}, p_{2}, \ldots, p_{n}, \ldots$ enumerate the prime numbers. This way, if $w_{i}$ is a proper prefix of $w_{j}$, then $w_{i}$ will be encoded as a lower number than $w_{j}$. Now enumerate the words $w_{i}$ in the order of their encodings.

In the rest of Section A, we assume such a topologically sorted indexing scheme $\left(w_{j}\right)_{j \in J}$ for $\mathcal{W}$.

Lemma 7. For every $n \in J, P_{g}\left(w_{n}\right)$ is concentrated on $\operatorname{Path}_{F}\left(w_{n}\right)$.

Proof. Since $Z$ and $\Lambda$ have measurable diagonals, so does $Z \Lambda^{*}$. For each $n \in J$, $\left\{w_{n}\right\}$ is therefore measurable in $Z \Lambda^{*}$, so we can apply Lemma 2 and conclude that $P_{g}\left(w_{n}\right)$ is concentrated on $\operatorname{Path}_{F}\left(w_{n}\right)$.

In particular, the restriction $P_{g}\left(w_{n}\right)_{\mid P a t h_{F}\left(w_{n}\right)}$ makes sense. To reduce visual clutter, we simply write $P_{g}\left(w_{n}\right)$ for the latter probability measure on $\operatorname{Path}_{F}\left(w_{n}\right)$.

We shall write $w_{n} \Lambda^{\mathbb{N}}$ for the set of words in $Z \Lambda^{\mathbb{N}}$ that have $w_{n}$ as a prefix. As the product $\left\{w_{n}\right\} \times \Lambda^{\mathbb{N}}$, this is a measurable set. (Recall that one-element sets are measurable, since $Z$ and $\Lambda$ have measurable diagonals.)

We wish to draw the values $w_{j} \omega_{j}$ of $\widetilde{f}\left(w_{j}\right), j \in J$, in a consistent way, namely, if we have already mapped $w_{i}$ to the value $w_{i} \omega_{i}$, and $w_{i} \preceq w_{j} \preceq w_{i} \omega_{i}$, then we have no choice and must choose to map $w_{j}$ to $w_{i} \omega_{i}$ as well. We achieve this by using Ionescu-Tulcea's Theorem 2 on another probabilistic transition system, defined as follows, and which we only use as a mathematical helper. This is certainly no real-life, practical transition system, and is not meant to be.

We let $Q_{0}=\{*\}, q_{0}=*, Q_{n}=\operatorname{Path}_{F}\left(w_{n}\right)$ for $n \in J$, and $g_{n}: \prod_{i=0}^{n-1} Q_{i} \rightarrow$ $\mathcal{P}\left(Q_{n}\right)$ map $\left(*, w_{1} \omega_{1}, \cdots, w_{n-1} \omega_{n-1}\right)$ to:

- the Dirac mass $\delta_{w_{i} \omega_{i}}$, where $i$ is the least index in $1, \cdots, n-1$ such that $w_{i} \preceq w_{n} \preceq w_{i} \omega_{i}$, if one such index exists; this implements consistency;

- otherwise, the probability measure $P_{g}\left(w_{n}\right)$.

Both are probability measures on $Q_{n}=\operatorname{Path}_{F}\left(w_{n}\right)$ : the second one by Lemma 7 , the first one because $w_{i} \omega_{i}$ is a path in $P a t h_{F}$ starting with $w_{n}$.

Lemma 8. For every $n \in J, g_{n}$ is measurable.

Proof. For every $i, 1 \leq i<n$, such that $w_{i} \preceq w_{n}$, the set $A_{i}$ of infinite words $w_{i} \omega_{i}$ in $\operatorname{Path}_{F}\left(w_{i}\right)$ that have $w_{n}$ as a prefix is $\operatorname{Path}_{F}\left(w_{i}\right) \cap w_{n} \Lambda^{\mathbb{N}}$, hence is measurable. By extension, when $w_{i} \npreceq w_{n}$, write $A_{i}$ for the empty set. Rephrasing the definition, $g_{n}\left(*, w_{1} \omega_{1}, \cdots, w_{n-1} \omega_{n-1}\right)$ is defined as $\mathfrak{e}_{\text {Path }_{F}\left(w_{n}\right)}\left(w_{i} \omega_{i}\right)$ on $E_{i}=$ $\{*\} \times \overline{A_{1}} \times \cdots \times \overline{A_{i-1}} \times A_{i} \times\left|Q_{i+1}\right| \times \cdots \times\left|Q_{n-1}\right|, 1 \leq i<n$ (recall that $\mathfrak{e}$ 
is the monad unit, which is a measurable map), and as $P_{g}\left(w_{n}\right)$ on $\overline{\bigcup_{i=1}^{n-1} E_{i}}$ (a constant map). So $g_{n}$ is a patch of measurable maps, and is therefore measurable by Lemma 4 .

We can now apply Ionescu-Tulcea's Theorem, as promised, and obtain a probability measure on $\{*\} \times \prod_{j \in J} \operatorname{Path}_{F}\left(w_{j}\right) \cong \prod_{j=1}^{+\infty} \operatorname{Path}_{F}\left(w_{j}\right)$ that we decide to call $\mu_{\mathcal{W}}$. (This is the measure $P_{g_{*}}(*)$ of Theorem 2, but we wish to avoid any visual confusion with $P_{g}$.)

Let $C s t_{\mathcal{W}}$ be the set of tuples of paths $\left(w_{j} \omega_{j}\right)_{j \in J}$ in $\prod_{j \in J} \operatorname{Path}_{F}\left(w_{j}\right)$ that are consistent, i.e., such that for all $i, j \in J$ such that $w_{i} \preceq w_{j} \preceq w_{i} \omega_{i}, w_{j} \omega_{j}=w_{i} \omega_{i}$. These are the only ones we can ever hope to produce from a pure scheduler $f$, namely, that are of the form $\left(\widetilde{f}\left(w_{j}\right)\right)_{j \in J}$.

Proposition 5. The probability measure $\mu_{\mathcal{W}}$ is concentrated on the set $C s t_{\mathcal{W}}$ of consistent tuples.

Proof. Let $\mathcal{F}_{n}$ be the multifunction from $\prod_{j=1}^{n-1} \operatorname{Path}_{F}\left(w_{j}\right)$ to $\operatorname{Path}_{F}\left(w_{n}\right)$ defined, similarly to $g_{n}$, by letting $\mathcal{F}_{n}\left(w_{1} \omega_{1}, \cdots, w_{n-1} \omega_{n-1}\right)$ be:

- $\left\{w_{i} \omega_{i}\right\}$, where $i$ is the least index in $1, \cdots, n-1$ such that $w_{i} \preceq w_{n} \preceq w_{i} \omega_{i}$, if one such index exists;

- otherwise, Path $_{F}\left(w_{n}\right)$.

One checks easily that $\mathcal{F}_{n}$ is a measurable multifunction, and that $g_{n}\left(w_{1} \omega_{1}, \cdots\right.$, $\left.w_{n-1} \omega_{n-1}\right)$ is concentrated on $\mathcal{F}_{n}\left(w_{1} \omega_{1}, \cdots, w_{n-1} \omega_{n-1}\right)$. We apply Lemma 2 to $\mathcal{E}_{n}=\operatorname{Gr} \mathcal{F}_{n}$ and obtain that $\mu_{\mathcal{W}}$ is supported on Path $_{\mathcal{E}}$.

It remains to show that $\operatorname{Path}_{\mathcal{E}}=C s t_{\mathcal{W}}$. Given any inconsistent tuple $\left(w_{j} \omega_{j}\right)_{j \in J}$, there must be two indices $i, j \in J$ such that $w_{i} \preceq w_{j} \preceq w_{i} \omega_{i}$ but $w_{j} \omega_{j} \neq w_{i} \omega_{i}$. Since $\mathcal{W}$ is topologically sorted, $i \leq j$. Take $i$ minimal. Then $w_{j} \omega_{j}$ would be outside $\left\{w_{i} \omega_{i}\right\}=\mathcal{F}_{j}\left(w_{1} \omega_{1}, \cdots, w_{j-1} \omega_{j-1}\right)=\left(\mathcal{E}_{j}\right)_{\mid\left(w_{1} \omega_{1}, \cdots, w_{j-1} \omega_{j-1}\right)}$, showing that the tuple is not in $\operatorname{Path}_{\mathcal{E}}$. This establishes that $\operatorname{Path}_{\mathcal{E}} \subseteq C s t_{\mathcal{W}}$. The converse inclusion is obvious.

The restriction $\mu_{\mathcal{W} \mid C s t_{\mathcal{W}}}$ therefore makes sense. Again, we simply write $\mu_{\mathcal{W}}$ for this restriction, and consider it as a probability measure on $C s t_{\mathcal{W}}$.

Given $w \in \mathcal{W}$, say $w=w_{j}$, let us write $[w: \mathcal{E}]$ for the set of consistent tuples $\overrightarrow{\mathbf{w} \omega}$ in $C s t_{\mathcal{W}}$ such that $\omega_{j} \in \mathcal{E}$.

A set $A$ of words in $Z \Lambda^{*}$ is prefix-closed if and only if, for every $w \in Z \Lambda^{*}$ and $\lambda \in \Lambda, w \lambda \in \mathcal{I}$ implies $w \in \mathcal{I}$.

Lemma 9. Let $\left\{w_{1}^{\prime}, \ldots, w_{n}^{\prime}\right\}$ be a finite prefix-closed set of words in $Z \Lambda^{*}$. For all measurable subsets $\mathcal{E}_{1}, \ldots, \mathcal{E}_{n}$ of $\Lambda^{\mathbb{N}}$, the value of $\mu_{\mathcal{W}}\left(\bigcap_{i=1}^{n}\left[w_{i}^{\prime}: \mathcal{E}_{i}\right]\right)$ is independent of the countably infinite set $\mathcal{W}$, provided it is a superset of $\left\{w_{1}^{\prime}, \ldots, w_{n}^{\prime}\right\}$.

Proof. Consider any countably infinite superset $\mathcal{W}$ of $\left\{w_{1}^{\prime}, \ldots, w_{n}^{\prime}\right\}$. Write $\mathcal{W}$ as $\left(w_{j}\right)_{j \in J}$, as usual. Let $w_{1}^{\prime}=w_{j_{1}}, \ldots, w_{n}^{\prime}=w_{j_{n}}$. Up to permutation, we may assume that $j_{1}<j_{2}<\cdots<j_{n}$. Then $\bigcap_{i=1}^{n}\left[w_{i}^{\prime}: \mathcal{E}_{i}\right]$ is equal to the intersection of $\operatorname{Cst}_{\mathcal{W}}$ with $\prod_{j=1}^{j_{1}-1} \operatorname{Path}_{F}\left(w_{j}\right) \times w_{j_{1}} \mathcal{E}_{1} \times \prod_{j=j_{1}+1}^{j_{2}-1} \operatorname{Path}_{F}\left(w_{j}\right) \times w_{j_{2}} \mathcal{E}_{2} \times \cdots \times$ 
$\prod_{j=j_{n-1}+1}^{j_{n}-1} \operatorname{Path}_{F}\left(w_{j}\right) \times w_{j_{n}} \mathcal{E}_{n} \times \prod_{j=j_{n}+1}^{+\infty} \operatorname{Path}_{F}\left(w_{j}\right)$. We now use formula (2). This requires an abbreviation for all the integrals $\int_{w_{j} \omega_{j} \in Z \Lambda^{\mathbb{N}}} d g_{j}\left(*, w_{1} \omega_{1}, \cdots\right.$, $\left.w_{j-1} \omega_{j-1}\right)$ with $j \notin\left\{j_{1}, j_{2}, \cdots, j_{n}\right\}$-which will turn to be useless: write $\iint_{k \cdots \ell} \overrightarrow{\mathbf{d g}}$ for the list of symbols

$$
\begin{array}{r}
\int_{w_{k} \omega_{k} \in \operatorname{Path}_{F}\left(w_{k}\right)} d g_{k}\left(*, w_{1} \omega_{1}, \cdots, w_{k-1} \omega_{k-1}\right) \\
\int_{w_{k+1} \omega_{k+1} \in \operatorname{Path}_{F}\left(w_{k+1}\right)} d g_{k+1}\left(*, w_{1} \omega_{1}, \cdots, w_{k} \omega_{k}\right) \\
\cdots \int_{w_{\ell} w_{\ell} \in \operatorname{Path}_{F}\left(w_{\ell}\right)} d g_{\ell}\left(*, w_{1} \omega_{1}, \cdots, w_{\ell-1} \omega_{\ell-1}\right) .
\end{array}
$$

We can now write:

$$
\begin{aligned}
\mu_{\mathcal{W}}\left(\bigcap_{i=1}^{n}\left[w_{i}^{\prime}: \mathcal{E}_{i}\right]\right)= & \iint_{1 \cdots j_{1}-1} \overrightarrow{\mathbf{d g}} \int_{w_{j_{1}} \omega_{j_{1}} \in w_{j_{1}} \mathcal{E}_{1}} d g_{j_{1}}\left(*, w_{1} \omega_{1}, \cdots, w_{j_{1}-1} \omega_{j_{1}-1}\right) \\
& \iint_{j_{1}+1 \cdots j_{2}-1} \overrightarrow{\mathbf{d g}} \int_{w_{j_{2}} \omega_{j_{2}} \in w_{j_{2}} \mathcal{E}_{2}} d g_{j_{2}}\left(*, w_{1} \omega_{1}, \cdots, w_{j_{2}-1} \omega_{j_{2}-1}\right) \cdots \\
& \iint_{j_{n-1}+1 \cdots j_{n}-1} \overrightarrow{\mathbf{d g}} \int_{w_{j_{n}} \omega_{j_{n}} \in w_{j_{n}} \mathcal{E}_{n}} d g_{j_{n}}\left(*, w_{1} \omega_{1}, \cdots, w_{j_{n}-1} \omega_{j_{n}-1}\right) .
\end{aligned}
$$

Since $\left\{w_{1}, \ldots, w_{n}\right\}$ is prefix-closed, for each $i, 1 \leq i \leq n, g_{j_{i}}\left(*, w_{1} \omega_{1}, \cdots\right.$, $\left.w_{j_{i}-1} \omega_{j_{i}-1}\right)$ can be written as a function $g_{i}^{\prime}\left(w_{j_{1}} \omega_{j_{1}}, \cdots, w_{j_{i-1}} \omega_{j_{i-1}}\right)$ of just those words $w_{k} \omega_{k}$ with $k \in\left\{j_{1}, \ldots, j_{n}\right\}, k<j_{i}$. Explicitly, $g_{i}^{\prime}\left(w_{j_{1}} \omega_{j_{1}}, \cdots, w_{j_{i-1}} \omega_{j_{i-1}}\right)$ is $\delta_{w_{j_{\ell}} \omega_{j_{\ell}}}$ where $\ell$ is the least index, $1 \leq \ell<i$, such that $w_{j_{\ell}} \preceq w_{j_{i}} \preceq w_{j_{\ell}} \omega_{j_{\ell}}$ if one exists, and $P_{g}\left(w_{j_{i}}\right)$ otherwise. In (4), the final integral $\int_{w_{j_{n}} \omega_{j_{n}} \in w_{j_{n}} \mathcal{E}_{n}} d g_{j_{n}}\left(*, w_{1} \omega_{1}\right.$, $\left.\cdots, w_{j_{n}-1} \omega_{j_{n}-1}\right)=\int_{w_{j_{n}} \omega_{j_{n}} \in w_{j_{n}} \mathcal{E}_{n}} d g_{n}^{\prime}\left(w_{j_{1}} \omega_{j_{1}}, \cdots, w_{j_{n-1}} \omega_{j_{n-1}}\right)$ is independent of all the formal variables $w_{j_{n-1}+1} \omega_{j_{n-1}+1}, \ldots, w_{j_{n}-1} \omega_{j_{n}-1}$ that the integrals $\int_{w_{j} \omega \in \operatorname{Path}_{F}\left(w_{j}\right)} d g_{j}\left(*, w_{1} \omega_{1}, \cdots, w_{j-1} \omega_{j-1}\right)$ hidden in $\iint_{j_{n-1}+1 \cdots j_{n}-1} \overrightarrow{\mathbf{d g}}$ introduce. Since all these integrals are with respect to probability measures, they merely contribute a factor of 1 . We repeat the process, from right to left in (4), erasing all the notations $\iint_{j_{n-1}+1 \cdots j_{n}-1} \overrightarrow{\mathbf{d g}}$, and obtain:

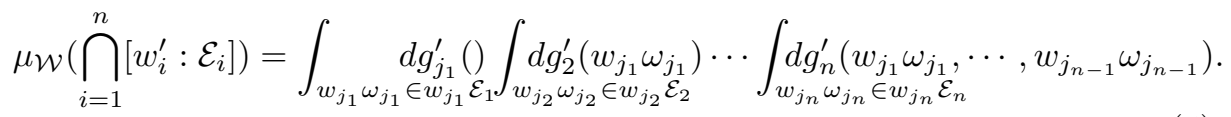

It is now evident that $\mu_{\mathcal{W}}\left(\bigcap_{i=1}^{n}\left[w_{i}^{\prime}: \mathcal{E}_{i}\right]\right)$ is independent of $\mathcal{W}$.

Applying (5) to the case $n=1, w_{j_{1}}=z \in Z$ (which is automatically prefixclosed), and noticing that $g_{j_{1}}^{\prime}()=P_{g}\left(w_{j_{1}}\right)=P_{g}(z)$, we obtain:

Lemma 10. For every $z \in Z$, for every countably infinite set $\mathcal{W}$ of words of $Z \Lambda^{*}$ containing $z$, for every measurable subset $\mathcal{E}$ of $\Lambda^{\mathbb{N}}, \mu_{\mathcal{W}}([z: \mathcal{E}])=P_{g}(z)(z \mathcal{E})$.

Proposition 6. For every consistent tuple $\overrightarrow{\mathbf{w} \omega}=\left(w_{j} \omega_{j}\right)_{j \in J}$ in Cst $t_{\mathcal{W}}$, there is a pure scheduler $f_{\overrightarrow{\mathbf{w} \omega}}$ such that $\widetilde{f}_{\overrightarrow{\mathbf{w} \omega}}\left(w_{j}\right)=w_{j} \omega_{j}$ for every $j \in J$. Moreover, the map $\alpha_{\mathcal{W}}: \overrightarrow{\mathbf{w} \omega} \in C s t_{\mathcal{W}} \mapsto f_{\overrightarrow{\mathbf{w} \omega}} \in \widehat{\operatorname{Sel}}(F)$ is measurable. 
Proof. If $w \in Z \Lambda^{*}$ is a prefix of an infinite word in $\Lambda^{\mathbb{N}}$, then this infinite word can be written in a unique way as $w \lambda \omega$ for some $\lambda \in \Lambda$ and $\omega \in \Lambda^{\mathbb{N}}$ : let us call $\lambda$ the letter after $w$ in the infinite word.

Since $(Z, \Lambda, F)$ is an IT automaton, $F$ has a measurable selection $\sigma$. For each consistent tuple $\overrightarrow{\mathbf{w} \omega}=\left(w_{j} \omega_{j}\right)_{j \in J}$ in $C s t_{\mathcal{W}}$, we define a pure scheduler $f_{\overrightarrow{\mathbf{w}} \vec{\omega}}$ as follows. For every $w \in Z \Lambda^{*}$,

- either $w_{j} \preceq w \preceq w_{j} \omega_{j}$ for some $j \in J$, and $f_{\overrightarrow{\mathrm{w}} \vec{\omega}}(w)$ is the letter after $w$ in $w_{j} \omega_{j}$

- or $w_{j} \preceq w \preceq w_{j} \omega_{j}$ for no $j \in J$, and $f_{\overline{\mathrm{w}} \vec{\omega}}(w)=\sigma(w)$.

In the first case, it does not matter which $j \in J$ is picked, because of consistency. Imagine indeed that $w_{i} \preceq w \preceq w_{i} \omega_{i}$ and $w_{j} \preceq w \preceq w_{j} \omega_{j}$ for two indices $i, j \in J$. Since $w_{i}$ and $w_{j}$ are two prefixes of the same word $w$, one of them must be a prefix of the other, say $w_{i} \preceq w_{j}$. Then $w_{j} \preceq w \preceq w_{i} \omega_{i}$, and consistency entails that $w_{i} \omega_{i}=w_{j} \omega_{j}$, so the letter after $w$ is the same in both infinite words.

For short, let us say that $w$ is stored in $\overrightarrow{\mathbf{w} \omega}$ if and only if $w_{j} \preceq w \preceq w_{j} \omega_{j}$ for some $j \in J$. In this case, it is easy to see that $\widetilde{f}_{\overrightarrow{\mathrm{w} \omega}}(w)=w_{j} \omega_{j}$. This implies our first claim, namely that $\widetilde{f}_{\overrightarrow{\mathrm{w}} \vec{\omega}}\left(w_{j}\right)=w_{j} \omega_{j}$ for every $j \in J$.

When $w$ is not stored in $\overrightarrow{\mathbf{w}} \vec{\omega}$, the situation is a bit more complicated. Let $\widetilde{\sigma}_{0}(w)=w, \widetilde{\sigma}_{k+1}(w)=\sigma_{k}(w) \sigma\left(\sigma_{k}(w)\right)$, be the ever longer sequence of finite prefixes of $\widetilde{\sigma}(w)$. If no $\widetilde{\sigma}_{k}(w)$ is stored in $\overrightarrow{\mathbf{w} \omega}$, then $\widetilde{f}_{\overrightarrow{\mathbf{w}} \vec{\omega}}(w)=\widetilde{\sigma}(w)$. But there may be a $k \in \mathbb{N}$ such that $\widetilde{\sigma}_{k}(w)$ is stored in $\overrightarrow{\mathbf{w} \omega}$. Taking the least such $k$, it must be the case that $\widetilde{f}_{\overrightarrow{\mathbf{w}} \vec{\omega}}(w)=w_{j} \omega_{j}$ where $j$ is any index of $J$ such that $w_{j} \preceq \widetilde{\sigma}_{k}(w) \preceq w_{j} \omega_{j}$. These remarks being made, let us proceed.

We wish to show that the map $\alpha_{\mathcal{W}}: \overrightarrow{\mathbf{w} \omega} \in C s t_{\mathcal{W}} \mapsto f_{\overrightarrow{\mathbf{w} \omega}} \in \widetilde{\operatorname{Sel}}(F)$ is measurable. For now, fix $w \in Z \Lambda^{*}$.

The set $S_{k}$ of tuples $\overrightarrow{\mathbf{w} \omega} \in C s t$ such that $\widetilde{\sigma}_{k}(w)$ is stored in $\overrightarrow{\mathbf{w}} \vec{\omega}$, is measurable. Indeed, $\widetilde{\sigma}_{k}(w)$ is stored in $\overrightarrow{\mathbf{w} \omega}$ if and only if there is a $j \in J$ such that $w_{j} \preceq$ $\widetilde{\sigma}_{k}(w) \preceq w_{j} \omega_{j}$, so $S_{k}=\bigcup_{w_{j} \preceq \widetilde{\sigma}_{k}(w)} \pi_{j}^{-1}\left(\widetilde{\sigma}_{k}(w) \Lambda^{\mathbb{N}}\right)$, where $\pi_{j}: C s t \rightarrow \operatorname{Path}_{F}\left(w_{i}\right)$ is projection onto the $j$ th component. It follows that the set $S_{=k}=S_{k} \backslash \bigcup_{\ell=0}^{k-1} S_{\ell}$ of tuples $\overrightarrow{\mathbf{w} \omega} \in C s t$ such that $k$ is the least natural number such that $\widetilde{\sigma}_{k}(w)$ is stored in $\overrightarrow{\mathbf{w} \omega}$ is also measurable. Let $S_{\infty}$ be the (measurable) complement of $\bigcup_{k=0}^{+\infty} S_{k}$.

Let $\varphi_{k}$ map each $\overrightarrow{\mathbf{w} \omega} \in S_{k}$ to $\widetilde{f}_{\overrightarrow{\mathbf{w}} \vec{\omega}}(w)$, i.e., to $w_{j} \omega_{j}$ where $j \in J$ is such that $w_{j} \preceq \widetilde{\sigma}_{k}(w) \preceq w_{j} \omega_{j}$. For every measurable subset $\mathcal{E}$ of $Z \Lambda^{\mathbb{N}}, \varphi_{k}^{-1}(\mathcal{E})=$ $\bigcup_{w_{j} \preceq \widetilde{\sigma}_{k}(w)} \widetilde{\sigma}_{k}(w) \Lambda^{\mathbb{N}}$ is measurable, so $\varphi_{k}$ is measurable. Also, the function $\varphi_{\infty}$ that maps each $\overrightarrow{\mathbf{w} \omega} \in S_{\infty}$ to $\widetilde{f}_{\overrightarrow{\mathbf{w}} \vec{\omega}}(w)=\widetilde{\sigma}(w)$ is measurable since constant. By patching $\varphi_{k}, k \in \mathbb{N}$, and $\varphi_{\infty}$ (Lemma 4), we obtain that the map $\alpha_{w}: \overrightarrow{\mathbf{w} \omega} \in$ $C s t \mapsto \widetilde{f}_{\overrightarrow{\mathrm{w}} \omega}(w)$ is measurable.

We now observe that, for all $w \in Z \Lambda^{*}$ and $\mathcal{E} \in \Sigma_{\Lambda^{\mathbb{N}}}, \alpha_{\mathcal{W}}^{-1}([w \widetilde{\mathcal{E}}])=$ $\left\{\overrightarrow{\mathbf{w} \omega} \in C s t_{\mathcal{W}} \mid \widetilde{f}_{\overrightarrow{\mathbf{w}} \vec{\omega}}(w) \in w \mathcal{E}\right\}=\alpha_{w}^{-1}(w \mathcal{E})$, which is measurable: so $\alpha_{\mathcal{W}}$ is measurable. 